Review

\title{
Cultivation, Genetic, Ethnopharmacology, Phytochemistry and Pharmacology of Moringa oleifera Leaves: An Overview
}

\author{
Alessandro Leone ${ }^{1,2, *}$, Alberto Spada ${ }^{3}$, Alberto Battezzati ${ }^{1,2}$, Alberto Schiraldi ${ }^{2}$, \\ Junior Aristil ${ }^{3}$ and Simona Bertoli ${ }^{1,2}$
}

1 International Center for the Assessment of Nutritional Status (ICANS), University of Milan, Via Sandro Botticelli 21, 20133 Milan, Italy; E-Mails: alberto.battezzati@unimi.it (A.B.); simona.bertoli@unimi.it (S.B.)

2 Department of Food, Environmental and Nutritional Sciences (DeFENS), University of Milan, Via Mangiagalli 25, 20133 Milan, Italy; E-Mail: alberto.schiraldi@unimi.it

3 Department of Agricultural and Environmental Sciences-Production, Landscape, Agroenergy (DISAA), University of Milan, Via Celoria 2, 20133 Milan, Italy; E-Mails: alberto.spada@unimi.it (A.S.); junior.aristil@unimi.it (J.A.)

* Author to whom correspondence should be addressed; E-Mail: alessandro.leone1@unimi.it; Tel.: +39-2-5031-6652; Fax: +39-2-5021-6077.

Academic Editor: Maurizio Battino

Received: 21 April 2015 / Accepted: 20 May 2015 / Published: 5 June 2015

\begin{abstract}
Moringa oleifera is an interesting plant for its use in bioactive compounds. In this manuscript, we review studies concerning the cultivation and production of moringa along with genetic diversity among different accessions and populations. Different methods of propagation, establishment and cultivation are discussed. Moringa oleifera shows diversity in many characters and extensive morphological variability, which may provide a resource for its improvement. Great genetic variability is present in the natural and cultivated accessions, but no collection of cultivated and wild accessions currently exists. A germplasm bank encompassing the genetic variability present in Moringa is needed to perform breeding programmes and develop elite varieties adapted to local conditions. Alimentary and medicinal uses of moringa are reviewed, alongside the production of biodiesel. Finally, being that the leaves are the most used part of the plant, their contents in terms of bioactive compounds and their pharmacological properties are discussed. Many studies conducted on cell lines and animals seem concordant in their support for these properties. However, there are still too few studies on humans to
\end{abstract}


recommend Moringa leaves as medication in the prevention or treatment of diseases. Therefore, further studies on humans are recommended.

Keywords: Moringa oleifera; ethnopharmacology; phytochemistry; pharmacology; diabetes; dislipidemia; cancer; genetic variability; molecular markers; breeding

\section{Origin and Geographical Distribution}

In the monogeneric genus Moringa of Moringaceae family there are 13 species (namely, M. arborea, indigenous to Kenya; M. rivae indigenous to Kenya and Ethiopia; M. borziana, indigenous to Somalia and Kenia; M. pygmaea indigenous to Somalia; M. longituba indigenous to Kenia, Ethiopia and Somalia; M. stenopetala indigenous to Kenya and Ethiopia; $M$ ruspoliana indigenous to Ethiopia; M. ovalifolia indigenous to Namibia and Angola; M. drouhardii, M. hildebrandi indigenous to Madagascar; M. peregrine indigenous o Red sea and Horn of Africa, M. concanensis, Moringa oleifera indigenous to sub-Himalayan tracts of Northern India [1]), among which Moringa oleifera (Figure 1) has so far become the most used and studied.

This species is a fast growing soft wood tree that can reach $12 \mathrm{~m}$ in height and is indigenous to the Himalayan foothills (northern India Pakistan and Nepal) [2,3]. Its multiple uses and potential attracted the attention of farmers and researchers in past historical eras. Ayurvedic traditional medicine says that Moringa oleifera can prevent 300 diseases and its leaves have been exploited both for preventive and curative purposes [4]. Moreover, a study in the Virudhunagar district of Tamil Nadu India reports Moringa among the species utilized by traditional Siddha healers [5]. Ancient Egyptians used Moringa oleifera oil for its cosmetic value and skin preparation [6]; even if the species never became popular among Greeks and Romans, they were aware of its medical properties [7]. Moringa oleifera has been grown and consumed in its original areas until recently (the 1990s) when a few researchers started to study its potential use in clarifying water treatments, while only later were its nutritional and medical properties "discovered" and the species was spread throughout almost all tropical countries. In 2001, the first international conference on Moringa oleifera was held in Tanzania and since then the number of congresses and studies increased disseminating the information about the incredible properties of Moringa oleifera. Now this species has been dubbed "miracle tree", or "natural gift", or "mother's best friend".

Moringa oleifera grows in any tropical and subtropical country with peculiar environmental features, namely, dry to moist tropical or subtropical clime, with annual precipitation of 760 to $2500 \mathrm{~mm}$ (it requires less than $800 \mathrm{~mm}$ irrigation) and temperature between 18 and $28{ }^{\circ} \mathrm{C}$. It grows in any soil type, but heavy clay and waterlogged, with $\mathrm{pH}$ between 4.5 and 8 , at an altitude up to $2000 \mathrm{~m}$ [8,9].

A study on local uses and geographical distribution of Moringa oleifera [10] that covers the major agro-ecological region in Nigeria, clearly established that "though considered a not indigenous species, Moringa oleifera has found wide acceptance among various ethnic Nigeria, who have exploited different uses (e.g., food, medicine, fodder etc.).

Nowadays, Moringa oleifera and its derivatives are distributed mainly in Middle East, African and Asian countries [11] and are still spreading to other areas. 


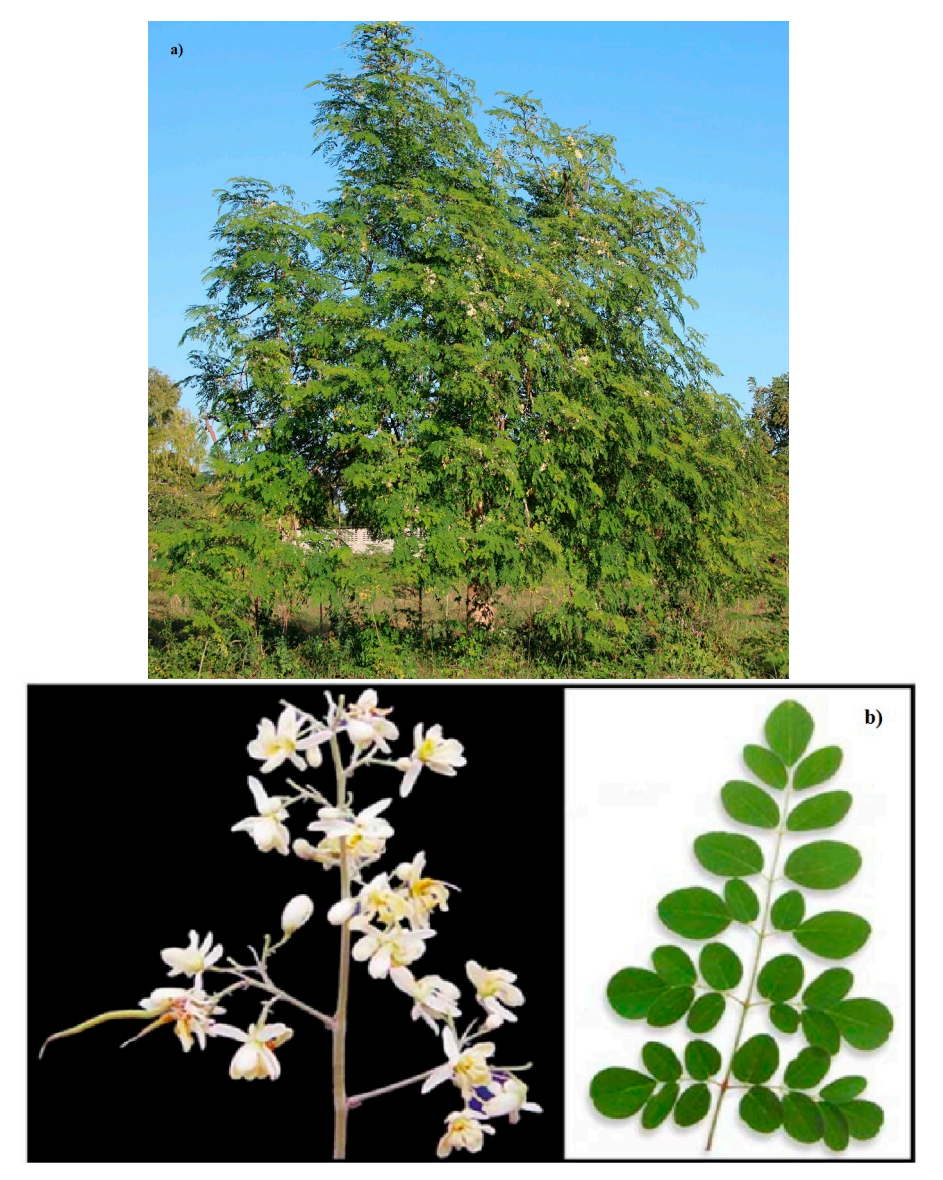

Figure 1. (a) A tree of Moringa oleifera; (b) Moringa flowers and leaves.

\section{Cultivation and Production}

Moringa oleifera development is achieved in two main ways: sowing and cutting.

Traditionally in Sudan the seeds are preferred while vegetative propagation is common in India, Indonesia and in some areas of West Africa [8].

Sowing requires selection of the seeds, when they are easily available and human labor is limited, while the possibility to transplant seedlings allows flexibility in field planting even if it requires extra labor and costs.

Seeds germinate within two weeks, at a maximum $2 \mathrm{~cm}$ depth. When sowing is planned in nursery, the seedlings can be transplanted when they reach about $30 \mathrm{~cm}$ (3-6 weeks after germination) [12].

The number of seeds per kilogram ranges from 3000 to 9000 , depending on the variety, with a germination rate of $80 \%-90 \%$ for ideal storage conditions $\left(3{ }^{\circ} \mathrm{C}, 5 \%-8 \%\right.$ moisture). However, the viability decreases if seeds remain at ambient temperature and high relative humidity, their germination rate dropping to $7.5 \%$ after three months $[3,13]$.

Cutting is preferred when seeds availability is scarce and/or when labor is not a limiting factor. Ramachandran et al. [14] reports that plants raised from seeds produce fruits of poorer quality, while Animashaun et al. [15] suggest that trees grown from seeds develop longer roots (an advantage for stabilization and access to water) compare to that grown from cuttings that have much shorter roots.

When hard woodcuttings (1-2 $\mathrm{m}$ long $4-16 \mathrm{~cm}$ diameter $[8,15])$ from adult trees are planted during the rainy season burying one third in the soil, they readily develop roots that in few months reach a 
considerable size [16]. Moringa oleifera is an exceptionally fast growing tree, in three months it can be $3 \mathrm{~m}$ high and in few years reaches $12 \mathrm{~m}$ if it is left to growth naturally. Since the tree vigorously re-sprouts after cutting, pruning or pollarding are usually practiced to enhance lateral branching and give the tree a bush shape in order to facilitate the harvest. Nevertheless, since literature reports about the good practice management of Moringa oleifera are scant, practical trials are needed [12]. Leaves and seeds are the parts of the plant of interest. Accordingly, the spatial distribution in planting Moringa oleifera trees is designed to facilitate the relevant harvest and the management practices.

For production of leaves, Moringa oleifera plantation can be designed as follows:

(i) intensive production with spacing ranging from $10 \mathrm{~cm} \times 10 \mathrm{~cm}$ to $20 \mathrm{~cm} \times 20 \mathrm{~cm}$, harvest interval between 35 to 45 days, irrigation and fertilization are needed;

(ii) semintensive production with spacing about $50 \mathrm{~cm} \times 100 \mathrm{~cm}$, harvest interval between 50 to 60 days, irrigation and fertilization suggested;

(iii) integrate in an agroforestry system with spacing distance of 2-4 m between rows, harvest interval around 60 days, fertilization and irrigation not strictly necessary.

Production decreases from intensive production to less dense spacing (agroforestry system), although a tremendous variability can be observed for a given spatial distribution and the same cultivation management. For example, the yield of an intensive plantation can range from 580 to $40 \mathrm{~m} / \mathrm{ha} /$ year [15], being season dependent with the largest yield in wet or cold season. There is a need for further studies to assess optimum spacing and harvest intervals that comply with the different climates and production systems [17-19]. Harvest can be mechanical or manual. Shoots are cut at a 0.5-1 $\mathrm{m}$ height above the ground; but leaves can be picked directly off the tree; this practice, however, albeit quicker, leads to a less vigorous re-growth.

For the production of seed a low density plantation has a positive effect on yields: typically $2.5 \times 2.5 \mathrm{~m}$ or $3 \times 3 \mathrm{~m}$ triangular pattern [20]. Fruits (trilobite capsule), referred as pods (brown color and dry and split longitudinally), ripen about three months after flowering and must be harvested as soon as possible. Each pod usually contains about $261-\mathrm{cm}$ diameter seeds lined by three whitish papery leaflets on the edge. Like for leaves, also the production of seed shows a tremendous variability. A single tree can produce from 15.000 to 25.000 seeds with an average weight of 0.3 gr per seed [21]; moreover early flowering varieties produce pods in six month, while other varieties require more than one year. After pruning, branches develop new pods within 6 months [1].

\section{Genetic and Breeding}

The major Moringa oleifera producer is India with an annual production of 1.1 to 1.3 million tons of tender fruits from an area of $380 \mathrm{~km}^{2}$ [22]. Information about the production in other countries is scarce. The great interest in Moringa oleifera does not concern its commercial value, being mainly related to its multipurpose uses and its ability to guarantee a reliable yield, while other crops cannot, in countries where people are mostly at risk of suffering from nutritional deficiencies. Indeed its cultivation is localized in developing countries where different parts of the plant are utilized: seeds for oil and water purification; leaves, seeds and fruits for their high nutritional value (nutritional integrator); leaves and seeds for biomass and animal feeding; different parts in traditional medicine. 
Moreover, Moringa has been planted around the world and is naturalized in many areas (i.e., almost the entire tropical belt) increasing the variability of the species.

As Moringa is a cross-pollinated tree, high heterogeneity in form and yield is expected. Several works indeed report variability in flowering time [23] (from annual type to perennial type), tree nature (from deciduous to evergreen), tree shape (from semi spread to upright), resistance to hairy caterpillar [22-24], flowering time (i.e., some tree flowering throughout the year while others flower in two distinct season) [14].

Although Moringa oleifera shows diversification in many characters and high morphological variability, which may become a resource for its improvement, the major factors that limit productivity are the absence of elite varieties adapted to local conditions and the use of seeds obtained through open pollination from plants in the planted area. Furthermore, despite the various uses of Moringa oleifera and its morphological differentiation, the number of accessions to collections and active germplasm banks are incipient across the world.

Many ecotypes are present in India: Jaffna (soft and taste fruits), Chavakacheri murungai (similar to Jaffna), Chemmurungai (red tipped fruits), Kadumurungai (small and inferior fruits) Palmurungai (bitter taste), Punamurungai (similar to Palmurungai), Kodikalmurungai (short fruit), Palmurungai, Puna Murungai and Kodikkal Murungai and wild Kadumurunga [14,25]. Recently two varieties (PKM-1; PKM2) have been developed at Horti Nursery Networks, Tamil Nadu, India, to improve pod production: usually those varieties are grown as annual; after two harvests the tree is dragged out and new seedlings are planted [26]. At Kerala Agricultural University (India) several varieties have been developed and available.

Outside India there are research centers focused on Moringa oleifera improvement across the world: AVDRC (Taiwan), Rural development initiative (Zambia), Moringa Philippines foundation (Philippines) Moringa community (Zambia).

In spite of the great variability of Moringa oleifera no institutions have a germplasm bank or data base with either cultivated or spontaneous accessions. The divergence between genetic variability inherent to the species and poor variability reflected in germplasm banks should be fixed since it represents an obstacle for the progress of breeding programs.

Moringa cytological studies revealed that Moringa oleifera has $2 \mathrm{c}$ genome size of $1.2 \mathrm{pg}$ [27] and it is a true diploid with $2 n=28$ [14]. Since 1999 molecular markers have become standards for the genetic characterization of Moringa oleifera. Studies started with the use of dominant markers until the development of co-dominant markers (in 2010) that allow distinction between homozygotes and heterozygotes, which provides optimal genetic information profiles.

Interestingly, out of 2857 scientific publications on Moringa oleifera in the primary database (Web of Science), only 12 include genetic characterization based on molecular markers. Furthermore, only 77 fragments of DNA and RNA sequences are available (data from NCBI nucleotide database). This means that the genetic approach and its potential application in breeding programs are just at the beginning step. The common aim of almost all the studies is the genetic diversification among different populations and/or accessions: dominant markers are the most used (66\% of all papers).

In spite of the limited range of dominant markers (heterozygote cannot be distinguished from homozygote specimens), the studies among commercial, cultivated or natural accessions have contributed to the understanding of genetic variability of Moringa oleifera. In this context, Amplified 
Fragment Length Polymorphism (AFLP) and Random Amplified Polymorphic DNA (RAPD) analyses along with Inter-Simple Sequence Repeat (ISSR) and cytochrome P450 were used. Muluvi et al. [28] used AFLP to investigate seven natural populations from India and introduced populations in Malawi and Kenya. Authors found a significant level of population differentiation and separation of genotypes based on geographical origin. Moreover, high portion of genetic variability was within Indian accessions. In line with these findings, the authors argued that Kenya populations presumably came from India, as suggested by the small number of genetically related accessions. Thank to the same molecular markers [29] the outcrossing rate in Moringa oleifera was detected: $26 \%$ of selfing in Moringa trees. This evidence had a strong impact on the breeding program, as inbreed lines and hybridization allowed improvement of the species.

RAPD were used by different authors to investigate cultivated and non-cultivated population of Tanzania [30], different accessions in Nigeria [31], accessions present in Embra Cosatal Teblelands Sergipe germplasm bank in Brazil [32], commercially grown varieties in India [33], new genotype developed in different countries (Thailand, USA, India and Malaysia, Tanzania, Taiwan) [34], and further accessions in Nigeria [35]. All these studies showed the higher level of genetic diversity in natural population with respect to the cultivated ones. Cultivated accessions present in the considered germplasm banks are genetically close and need to be widen to promote increased diversity and used in breeding programs. Many studies disagree with Muluvi's conclusions that a significant level of population differentiation and separation of genotypes can be based on the geographical origin. Indeed no clusters were found according to geographical origins. This could be due to the planting spread that produced a high rate of gene flow through cross-pollination. Interestingly, Popoola et al. [35] investigated morpho-metric characters along with molecular markers and showed a good correlation between 100 seeds weight with pod length, pod weight, number of seeds per lobule and number of seed per pod.

Studies on Moringa oleifera with co-dominat markers started in 2010 when Wu et al. [36] developed microsatellite markers. The first successful estimates of genetic diversity and population structure were obtained with Simple Sequence Repeat (SSR) in 2013 by Shahazad et al. [37]. These authors evaluated accessions collected in different locations of Pakistan and accessions from different countries (India, Tanzania, Senegal, Mozambique, Zimbabwe, Florida, Mexico, Haiti, Belize) obtained from Educational Concerns foe Hunger Organization (ECHO). They found high genetic diversity in wild Pakistan accessions, whereas low genetic diversity in ECHO accessions. Moreover, ECHO accessions are more similar to those of a single province of Pakistan. Most likely, British colonialists introduced Moringa oleifera in early of twentieth century in Africa from India, while in the 1784 an Englishman took Moringa oleifera over to Jamaica [37]. The export pathway was restricted to Indian coastal region (where most movement of goods and people took place) and involved a relatively small number of accessions that belonged to a common or few populations. This explains the low genetic variability within ECHO accessions with respect to the Pakistan ones.

Later on, a further investigation on twelve Indian populations, from northern and southern regions of India, was performed through SSR together with morphological markers [38]. In this study too, individuals from various geographical areas were not significantly different genetically, while a large variability exist in Indian populations. Morphological analysis on fourteen quantitative and eleven qualitative characters showed correlation among some quantitative characters, e.g., between tree tallness with fruit girth, trunk girth with tree branching. More SSR were identified in 2014 thanks to 
EST examination involving several plant species [39] and not utilized so far in Moringa oleifera genetic investigation.

Even if all the reported studies are valuables and have a tremendous importance for conservation, selection and collection of Moringa oleifera seeds, same questions are still to be addresses in order to develop an improved Moringa oleifera cultivation. Considering the cultivation challenges, some research activities should be prioritized: (i) collection and characterization of world accessions both cultivated and natural in order to obtain a true understanding of the genetic diversity and structure of Moringa oleifera; (ii) set a collaborative network among National and International Research Centre, O.N.G, farmers that already work on Moringa oleifera.

This will help scientists and producers to:

- have a reliable access to information about genetics and materials to develop better Moringa varieties and technologies for farming practices: phenotypic characterization is a priority to evaluated the accessions;

- ensure that Moringa production is improved along with best cultivation practice;

- focus research on the association between phenotypic and molecular data within the contest of breeding;

- define maps (both association map and physical map) to identify genes that may confer resistance to biotic and abiotic stress and quantitative traits loci (QTL) for a possible introgression of genes into commercial and cultivated accessions.

Today next generation sequencing (NGS) [40] is an approachable tool to discovery genome-wide genetic markers. This technique could be applied to species with no existing genome data like Moringa. Thank to NGS a saturated genetic map could be obtain within reasonable cost and time, in turn interesting characters could be identified and exploited in breeding programmes.

\section{Traditional Uses}

All plant parts of Moringa oleifera are traditionally used for different purposes, but leaves are generally the most used [10,41]. In particular, they are used in human and animal nutrition and in the traditional medicine. Leaves are rich in protein, mineral, beta-carotene and antioxidant compounds, which are often lacking among the populations of underdeveloped or developing countries. Moringa leaves are added to food preparations as integrators of the diet. In traditional medicine, these leaves are used to treat several ailments including malaria, typhoid fever, parasitic diseases, arthritis, swellings, cuts, diseases of the skin, genito-urinary ailments, hypertension and diabetes. They are also used to elicit lactation and boost the immune system (to treat HIV/AIDS related symptoms) [10,41-45], as well as cardiac stimulants and contraceptive remedy. One can directly consume either raw and dried leaves or the extract of an aqueous infusion.

Similarly, the use of seeds concerns both human nutrition and traditional medicine. Barks are boiled in water and soaked in alcohol to obtained drinks and infusions that can be used to treat stomach ailments (ease stomach pain, ulcer and aiding digestion), poor vision, joint pain, diabetes, anemia and hypertension [10,43], toothache, hemorrhoids, uterine disorder [10,44]. In a well known practice, Moringa seeds are used to sediment impurities of water [10]. 
Roots are soaked in water or alcohol and boiled with other herbs to obtained drinks and infusions as remedies for toothache, as anthelmintic and antiparalytic [10,41,42] drugs and as sex enhancers.

Finally, flowers are used to produce aphrodisiac substances and to treat inflammations, muscle diseases, hysteria, tumors and enlargement of the spleen $[42,44]$.

\section{Non Food or Medicinal Uses}

Beyond the uses of Moringa as a food and for human health, other possible uses exist. It can be used as a natural plant growth enhancer; indeed leaves are rich in zeatin (a plant hormone belong to the cytokinin group). Leaf extracts can stimulate plant growth and increasing crop yield. Researches performed using a spray based on leaf extracts of wheat, maize and rice support the wide range of beneficial effect on crops [46].

Moringa seed powder can be used for water purification, replacing dangerous and expensive chemicals such as aluminum sulfate [10].

Interestingly, leaf extracts and also seed extracts show biopesticide activity, effective against larvae and adults of Trigoderma granarium and can reduce the incidence of fungi on groundnut seeds [46].

One of the interesting applications of Moringa seeds is their utilization as biomass for biodiesel production.

Due to the increasing energy demand and environmental problems associated with fossil fuels, the improvement of alternative fuels and renewable sources of energy is required. Biodiesel can replace petroleum-derived oil (petrodiesel), without any sulphur or aromatic compound and with lower emission of monoxides, hydrocarbons and particulates. Furthermore, biodiesel can reduce dependence on imported fuels: a crucial problem in developing countries [47].

Moringa seeds have an oil content of $30 \%-40 \%$, with a high-quality fatty acid composition i.e., high oleic acid (>70\%) [48]. In addition they posses significant resistance to oxidative degradation. These proprieties make Moringa oil a good candidate to produce biodiesel after transesterificaton [48-50]. Biswas and John [51], in a study conducted in Australia, report that approximately $3030 \mathrm{~kg}$ of oil are required to produce 1000 liters of biodiesel. Furthermore, an equivalent of 3.03 tonnes/ha of oil seeds can be harvested from dry land, and 6.06 tonnes/ha can be harvested from irrigated land. Since biodiesel production with Moringa seed oil is a second generation production (i.e., not in direct competition with existing farmland and with food crops) and as Moringa can grown on degraded land, studies suggest that Moringa biodiesel is an acceptable substitute to fossil fuels, even when compared against biodiesel derived from vegetable oil of other species.

\section{Phytochemistry}

As Moringa oleifera leaves are most used part of the plant, we review articles concerning phytochemistry and pharmacological properties of leaves.

Several bioactive compounds were recognized in the leaves of Moringa oleifera. They are grouped as vitamins, carotenoids, polyphenol, phenolic acids, flavonoids, alkaloids, glucosinolates, isothiocyanates, tannins, saponins and oxalates and phytates (Figure 2). The amounts of different bioactive compounds found in Moringa oleifera leaves and reported in literature are summarized in Tables 1-9. 


\section{Vitamins}<smiles>CC1=C(/C=C/C(C)=C/C=C/C(C)=C/CO)C(C)(C)CCC1</smiles><smiles>CC(C)=C/C(C)=C/C(C)=C/C(C)=C/C=C/C(C)=C/C=C/C(C)=C/C=C/C=C(C)/C=C/C=C(C)/C=C/C1=C(C)CCCC1(C)C</smiles><smiles>O=C1OC(C(O)CO)C(O)=C1O</smiles>

L-Ascorbic acid<smiles>Cc1cc2nc3c(=O)[nH]c(=O)nc-3n(C[C@H](O)[C@H](O)[C@H](O)CO)c2cc1C</smiles>

\section{Flavonoids}<smiles>COc1cc(-c2oc3cc(O)cc(O)c3c(=O)c2O)cc(O)c1O</smiles><smiles>COc1cc(-c2oc3cc(O)cc(O)c3c(=O)c2O)ccc1O</smiles>

\section{Phenolic acids}<smiles>O=C(O)/C=C/c1ccc(O)c(O)c1</smiles><smiles>O=c1oc2c(O)c(O)cc3c(=O)oc4c(O)c(O)cc1c4c23</smiles>

Ellagic acid<smiles>O=c1c(O)c(-c2ccc(O)c(O)c2)oc2cc(O)cc(O)c12</smiles>

Quercetin

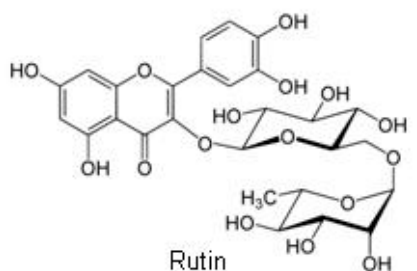

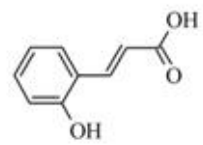

o-Coumaric acid

Chlorogenic acid<smiles>COc1cc(/C=C/C(=O)O)ccc1O</smiles>

Ferulic acid<smiles>O=C(O)c1cc(O)c(O)c(O)c1</smiles>

Gallic acid

Figure 2. Cont. 


\section{Alkaloids}

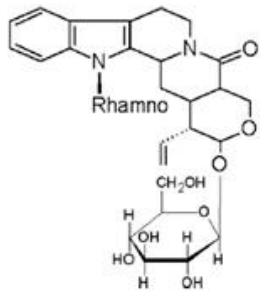

$N, \alpha$-L-rhamnopyranosyl vincosamide

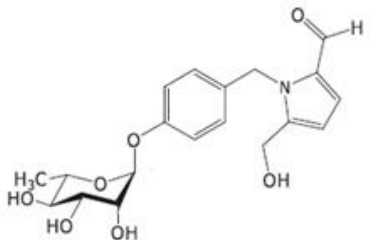

Pyrrolemarumine 4"-O- $\alpha$-L-rhamnopyranoside

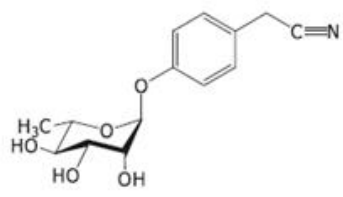

4-(a-L-rhamnopyranosyloxy)phenylacetonitrile (Niazirin)

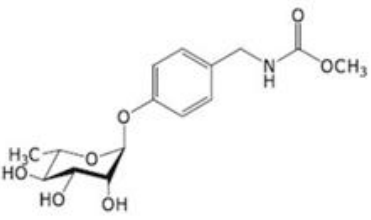

methyl 4-( $\alpha$-L-rhamnopy ranosyloxy)-benzylcarbamate<smiles>NC(=O)Cc1ccc(OC2C(C(=O)O)CCCC2C(=O)O)cc1</smiles>

4'-hydroxyphenylethanamide- $\alpha$-L-rhamnopyranoside (Marumoside $A)(R=H)$

4'-hydroxyphenylethanamide- $\alpha$ - $L$-rhamnopyranoside (Marumoside $B)(R=D$-Glucose $)$

\section{Glucosinolates}

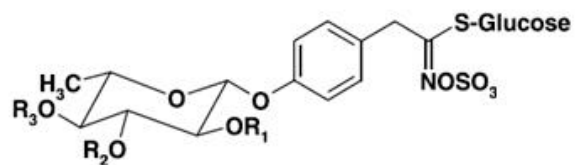

4-O-( $\alpha$-L-rhamnopyranosyloxy)-benzyl glucosinolate (R1, R2, R3 = H)

4-O-( $\alpha$-L-acetylrhamnopyranosyloxy)-benzyl glucosinolate Isomer $1\left(R 1, R 2=H_{;} R 3=A c\right)$

4-O-( $\alpha$-L-acetylrhamnopyranosyloxy)-benzyl glucosinolate Isomer $2\left(R 1, R 3=H_{;} R 2=A c\right)$

4-O-(o-L-acetylrhamnopyranosyloxy)-benzyl glucosinolate Isomer $3(R 2, R 3=H ; R 1=A c)$

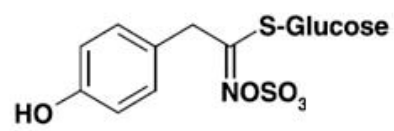

4-hydroxybenzyl glucosinolate (sinalbin)

\section{Isothiocyanates}

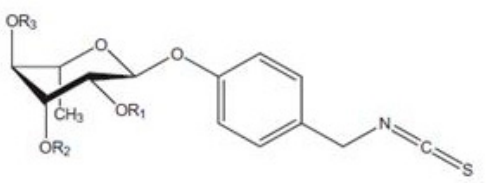

4-(o-L-rhamnosyloxy)benzyl isothiocyanate $(R 1, R 2, R 3=H)$

4-(4'-O-acetyl- $\alpha$-L-rhamnosyloxy)benzyl isothiocyanate $(R 1, R 2=H ; R 3=A c)$

4-(3'-O-acetyl- $\alpha$-L-rhamnosyloxy)benzyl isothiocyanate $(R 1, R 3=H ; R 2=A c)$

4-(2'-O-acetyl-a-L-rhamnosyloxy)benzyl isothiocyanate $\left(\mathrm{R} 2, \mathrm{R} 3=\mathrm{H}_{;} \mathrm{R} 1=\mathrm{Ac}\right)$

Figure 2. Chemical structure of bioactive compounds found in Moringa oleifera leaves. 
Table 1. Vitamins content in Moringa oleifera leaves.

\begin{tabular}{|c|c|c|c|c|c|c|c|c|}
\hline Bioactive Compound & Leaves & $\begin{array}{l}\text { Value Found } \\
\text { in Literature }\end{array}$ & $\begin{array}{c}\text { Value Express as } \\
\text { Dry Weight }\end{array}$ & Drying Method & Extractive Method & Analytical Method & Country & Reference \\
\hline \multicolumn{9}{|l|}{ Vitamins } \\
\hline \multirow{2}{*}{ Vitamin A } & fresh & $11,300 \mathrm{IU}$ & 45,200 IU & & $\mathrm{N} / \mathrm{A}$ & $\mathrm{N} / \mathrm{A}$ & India & [14] \\
\hline & fresh & $23,000 \mathrm{IU}$ & $92,000 \mathrm{IU}^{\mathrm{a}}$ & & $\mathrm{N} / \mathrm{A}$ & $\mathrm{N} / \mathrm{A}$ & Brazil & {$[52]$} \\
\hline \multirow{4}{*}{ Vitamin B1-Thiamine } & fresh & $0.06 \mathrm{mg} / 100 \mathrm{~g}$ & $0.24 \mathrm{mg} / 100 \mathrm{~g}$ & & N/A & $\mathrm{N} / \mathrm{A}$ & India & [14] \\
\hline & fresh & $0.21 \mathrm{mg} / 100 \mathrm{~g}$ & $0.84 \mathrm{mg} / 100 \mathrm{~g}$ & & N/A & N/A & N/A & [53] \\
\hline & fresh & $0.6 \mathrm{mg} / 100 \mathrm{~g}$ & $2.58 \mathrm{mg} / 100 \mathrm{~g}$ & & $\mathrm{~N} / \mathrm{A}$ & Microbiological method & India & [54] \\
\hline & dried & $2.64 \mathrm{mg} / 100 \mathrm{~g}$ & $2.85 \mathrm{mg} / 100 \mathrm{~g}$ & N/A & $\mathrm{N} / \mathrm{A}$ & $\mathrm{N} / \mathrm{A}$ & N/A & [53] \\
\hline \multirow{4}{*}{ Vitamin B2-Riboflavin } & fresh & $0.05 \mathrm{mg} / 100 \mathrm{~g}$ & $0.20 \mathrm{mg} / 100 \mathrm{~g}$ & & N/A & $\mathrm{N} / \mathrm{A}$ & India & {$[14]$} \\
\hline & fresh & $0.05 \mathrm{mg} / 100 \mathrm{~g}$ & $0.20 \mathrm{mg} / 100 \mathrm{~g}$ & & $\mathrm{~N} / \mathrm{A}$ & N/A & N/A & [53] \\
\hline & fresh & $0.17 \mathrm{mg} / 100 \mathrm{~g}$ & $0.726 \mathrm{mg} / 100 \mathrm{~g}$ & & $\mathrm{~N} / \mathrm{A}$ & Microbiological method & India & [54] \\
\hline & dried & $20.5 \mathrm{mg} / 100 \mathrm{~g}$ & $22.16 \mathrm{mg} / 100 \mathrm{~g}$ & $\mathrm{~N} / \mathrm{A}$ & N/A & $\mathrm{N} / \mathrm{A}$ & $\mathrm{N} / \mathrm{A}$ & [53] \\
\hline \multirow{4}{*}{ Vitamin B3-Niacin } & fresh & $0.8 \mathrm{mg} / 100 \mathrm{~g}$ & $3.20 \mathrm{mg} / 100 \mathrm{~g}$ & & N/A & N/A & India & {$[14]$} \\
\hline & fresh & $0.8 \mathrm{mg} / 100 \mathrm{~g}$ & $3.20 \mathrm{mg} / 100 \mathrm{~g}$ & & $\mathrm{~N} / \mathrm{A}$ & $\mathrm{N} / \mathrm{A}$ & $\mathrm{N} / \mathrm{A}$ & [53] \\
\hline & fresh & $0.82 \mathrm{mg} / 100 \mathrm{~g}$ & $3.5 \mathrm{mg} / 100 \mathrm{~g}$ & & $\mathrm{~N} / \mathrm{A}$ & Microbiological method & India & [54] \\
\hline & dried & $8.2 \mathrm{mg} / 100 \mathrm{~g}$ & $8.86 \mathrm{mg} / 100 \mathrm{~g}$ & $\mathrm{~N} / \mathrm{A}$ & $\mathrm{N} / \mathrm{A}$ & N/A & N/A & [53] \\
\hline \multirow{8}{*}{ Vitamin C-Ascorbic acid } & fresh & $220 \mathrm{mg} / 100 \mathrm{~g}$ & $880 \mathrm{mg} / 100 \mathrm{~g}$ & & $\mathrm{~N} / \mathrm{A}$ & $\mathrm{N} / \mathrm{A}$ & India & {$[14]$} \\
\hline & dried & $17.3 \mathrm{mg} / 100 \mathrm{~g}$ & $18.7 \mathrm{mg} / 100 \mathrm{~g}$ & N/A & N/A & N/A & $\mathrm{N} / \mathrm{A}$ & [53] \\
\hline & & $92 \mathrm{mg} / 100 \mathrm{~g}$ & $92 \mathrm{mg} / 100 \mathrm{~g}$ & Sun-drying for 4 days & \multirow{3}{*}{ N/A } & \multirow{3}{*}{ AOAC 2004} & \multirow{3}{*}{ India } & \multirow{3}{*}[55]{} \\
\hline & dried & $140 \mathrm{mg} / 100 \mathrm{~g}$ & $140 \mathrm{mg} / 100 \mathrm{~g}$ & Shadow-drying for 6 days & & & & \\
\hline & & $56 \mathrm{mg} / 100 \mathrm{~g}$ & $56 \mathrm{mg} / 100 \mathrm{~g}$ & Oven-drying at $60^{\circ} \mathrm{C}$ for $1 \mathrm{~h}$ & & & & \\
\hline & dried & $38.8 \mathrm{mg} / 100 \mathrm{~g}^{\mathrm{b}}$ & $38.8 \mathrm{mg} / 100 \mathrm{~g}^{\mathrm{b}}$ & Air-drying & Metaphosphoric acid & Indophenol titration & Pakistan & {$[56]$} \\
\hline & freeze-dried & $271 \mathrm{mg} / 100 \mathrm{~g}$ & $271 \mathrm{mg} / 100 \mathrm{~g}$ & Freeze-drying & Deionized water & Colorimetric method & Florida, USA & {$[57]$} \\
\hline & freeze-dried & $\begin{array}{l}920 \mathrm{mg} / 100 \mathrm{~g} \\
840 \mathrm{mg} / 100 \mathrm{~g} \\
680 \mathrm{mg} / 100 \mathrm{~g} \\
\end{array}$ & $\begin{array}{l}920 \mathrm{mg} / 100 \mathrm{~g} \\
840 \mathrm{mg} / 100 \mathrm{~g} \\
680 \mathrm{mg} / 100 \mathrm{~g} \\
\end{array}$ & Freeze-drying & $6 \%$ metaphosphoric acid & $\begin{array}{c}\text { Titration against 2,6- } \\
\text { dichlorophenolindophenol }\end{array}$ & $\begin{array}{c}\text { Nicaragua } \\
\text { India } \\
\text { Niger } \\
\end{array}$ & [58] \\
\hline \multirow{4}{*}{ Vitamin E-Tocopherol } & fresh & $9.0 \mathrm{mg} / 100 \mathrm{~g}$ & $16.21 \mathrm{mg} / 100 \mathrm{~g}$ & & N-hexane + ethyl acetate + BHT & Reverse-phase HPLC & Malaysia & [59] \\
\hline & dried & $113 \mathrm{mg} / 100 \mathrm{~g}$ & $122.16 \mathrm{mg} / 100 \mathrm{~g}$ & $\mathrm{~N} / \mathrm{A}$ & $\mathrm{N} / \mathrm{A}$ & N/A & $\mathrm{N} / \mathrm{A}$ & [53] \\
\hline & dried & $74.45 \mathrm{mg} / 100 \mathrm{~g}$ & $74.45 \mathrm{mg} / 100 \mathrm{~g}$ & Drying at $60^{\circ} \mathrm{C}$ for $8 \mathrm{~h}$ & $\begin{array}{c}\text { Microscale saponification and } \\
\text { extraction with n-hexane }\end{array}$ & HPLC & Mexico & {$[60]$} \\
\hline & dried & $77.0 \mathrm{mg} / 100 \mathrm{~g}$ & $85.08 \mathrm{mg} / 100 \mathrm{~g}$ & Air-dried under shade & $\mathrm{N} / \mathrm{A}$ & HPLC Fluorescence & South Africa & {$[61]$} \\
\hline
\end{tabular}

Abbreviations: ${ }^{\text {a }}$ Obtained considering a moisture of $75 \% ;{ }^{b}$ Mean value of samples collected in different seasons; N/A $=$ Not available. 
Table 2. Carotenoids content in Moringa oleifera leaves.

\begin{tabular}{|c|c|c|c|c|c|c|c|c|}
\hline $\begin{array}{c}\text { Bioactive } \\
\text { Compound }\end{array}$ & Leaves & Value Found in Literature & $\begin{array}{l}\text { Value Express } \\
\text { as Dry Weight }\end{array}$ & Drying Method & Extractive Method & $\begin{array}{c}\text { Analytical } \\
\text { Method }\end{array}$ & Country & Reference \\
\hline \multicolumn{9}{|l|}{ Carotenoids } \\
\hline \multirow{6}{*}{$\beta$-carotene } & fresh & $6.63 \mathrm{mg} / 100 \mathrm{~g}$ & $33.48 \mathrm{mg} / 100 \mathrm{~g}$ & & Acetone-n-hexane & HPLC & Taiwan & {$[62]$} \\
\hline & fresh & $6.8 \mathrm{mg} / 100 \mathrm{~g}$ & $27.22 \mathrm{mg} / 100 \mathrm{~g}$ & & $\mathrm{~N} / \mathrm{A}$ & $\mathrm{N} / \mathrm{A}$ & N/A & [53] \\
\hline & dried & $\begin{array}{c}36 \mathrm{mg} / 100 \mathrm{~g} \\
39.6 \mathrm{mg} / 100 \mathrm{~g} \\
37.8 \mathrm{mg} / 100 \mathrm{~g}\end{array}$ & $\begin{array}{c}36 \mathrm{mg} / 100 \mathrm{~g} \\
39.6 \mathrm{mg} / 100 \mathrm{~g} \\
37.8 \mathrm{mg} / 100 \mathrm{~g}\end{array}$ & $\begin{array}{c}\text { Sun-drying for } 4 \text { days } \\
\text { Shadow-drying for } 6 \text { days } \\
\text { Oven-drying at } 60^{\circ} \mathrm{C} \text { for } 1 \mathrm{~h}\end{array}$ & N/A & $\begin{array}{c}\text { AOAC } \\
2004\end{array}$ & India & {$[55]$} \\
\hline & dried & $16.3 \mathrm{mg} / 100 \mathrm{~g}$ & $17.62 \mathrm{mg} / 100 \mathrm{~g}$ & N/A & N/A & $\mathrm{N} / \mathrm{A}$ & N/A & [53] \\
\hline & dried & $18.5 \mathrm{mg} / 100 \mathrm{~g}$ & $20.44 \mathrm{mg} / 100 \mathrm{~g}$ & Air-dried under shade & $\mathrm{N} / \mathrm{A}$ & HPLC & South Africa & [61] \\
\hline & freeze-dried & $66 \mathrm{mg} / 100 \mathrm{~g}$ & $66 \mathrm{mg} / 100 \mathrm{~g}$ & Freeze-drying & Acetone & HPLC & Florida, USA & [57] \\
\hline \multirow{2}{*}{ Lutein } & fresh & $6.94 \mathrm{mg} / 100 \mathrm{~g}$ & $35.05 \mathrm{mg} / 100 \mathrm{~g}$ & & Acetone-n-hexane & HPLC & Taiwan & [62] \\
\hline & freeze-dried & $102 \mathrm{mg} / 100 \mathrm{~g}$ & $102 \mathrm{mg} / 100 \mathrm{~g}$ & Freeze-drying & Acetone & HPLC & Florida, USA & [57] \\
\hline
\end{tabular}

Abbreviation: N/A = Not available. 
Table 3. Polyphenols content in Moringa oleifera leaves.

\begin{tabular}{|c|c|c|c|c|c|c|c|c|}
\hline $\begin{array}{c}\text { Bioactive } \\
\text { Compound }\end{array}$ & Leaves & Value Found in Literature & $\begin{array}{c}\text { Value Express as } \\
\text { Dry Weight }\end{array}$ & Drying Method & Extractive Method & Analytical Method & Country & Reference \\
\hline \multicolumn{9}{|l|}{ Polyphenols } \\
\hline \multirow{24}{*}{ Total phenols } & \multirow{2}{*}{ dried } & $4581 \mathrm{mgGAE} / 100 \mathrm{~g}^{\mathrm{a}}$ & $4581 \mathrm{mgGAE} / 100 \mathrm{~g}^{\mathrm{a}}$ & \multirow{2}{*}{ Shade-drying } & \multirow{2}{*}{ Water Soxhlet extraction for $18-20 \mathrm{~h}$} & \multirow{2}{*}{ Folin-Ciocalteau } & \multirow{2}{*}{ India } & \multirow{2}{*}[63]{} \\
\hline & & $3602 \mathrm{mgGAE} / 100 \mathrm{~g}^{\mathrm{b}}$ & $3602 \mathrm{mgGAE} / 100 \mathrm{~g}^{\mathrm{b}}$ & & & & & \\
\hline & dried & $3290 \mathrm{mgGAE} / 100 \mathrm{~g}$ & $3290 \mathrm{mgGAE} / 100 \mathrm{~g}$ & $\mathrm{~N} / \mathrm{A}$ & $50 \% \mathrm{MeOH}$ & Folin-Ciocalteau & India & [64] \\
\hline & dried & $2090 \mathrm{mgGAE} / 100 \mathrm{~g}$ & $2090 \mathrm{mgGAE} / 100 \mathrm{~g}$ & $\mathrm{~N} / \mathrm{A}$ & $50 \% \mathrm{MeOH}, 100 \% \mathrm{MeOH}$ and water & Folin-Ciocalteau & India & [65] \\
\hline & dried & $10,504 \mathrm{mgGAE} / 100 \mathrm{~g}$ & $10,504 \mathrm{mgGAE} / 100 \mathrm{~g}$ & $\mathrm{~N} / \mathrm{A}$ & Water at $80^{\circ} \mathrm{C}$ for $2 \mathrm{~h}$ & Folin-Ciocalteau & India & [66] \\
\hline & dried & $10,616 \mathrm{mgGAE} / 100 \mathrm{~g}^{\mathrm{c}}$ & $10,616 \mathrm{mgGAE} / 100 \mathrm{~g}^{\mathrm{c}}$ & Air-drying & $80 \% \mathrm{MeOH}$ & Folin-Ciocalteau & Pakistan & {$[56]$} \\
\hline & \multirow{4}{*}{ dried } & $10,300 \mathrm{mgGAE} / 100 \mathrm{~g}$ & $10,300 \mathrm{mgGAE} / 100 \mathrm{~g}$ & \multirow{4}{*}{ Air-drying } & \multirow{3}{*}{ Extraction by shaker } & \multirow{4}{*}{ Folin-Ciocalteau } & \multirow{4}{*}{ Pakistan } & \multirow{4}{*}[67]{} \\
\hline & & $12,200 \mathrm{mgGAE} / 100 \mathrm{~g}$ & $12,200 \mathrm{mgGAE} / 100 \mathrm{~g}$ & & & & & \\
\hline & & $9720 \mathrm{mgGAE} / 100 \mathrm{~g}$ & $9720 \mathrm{mgGAE} / 100 \mathrm{~g}$ & & & & & \\
\hline & & $11,600 \mathrm{mgGAE} / 100 \mathrm{~g}$ & $11,600 \mathrm{mgGAE} / 100 \mathrm{~g}$ & & $80 \% \mathrm{EtOH}$ & & & \\
\hline & \multirow{4}{*}{ dried } & $9630 \mathrm{mgGAE} / 100 \mathrm{~g}$ & $9630 \mathrm{mgGAE} / 100 \mathrm{~g}$ & \multirow{4}{*}{ Air-drying } & \multirow{4}{*}{ Extraction by reflux } & \multirow{4}{*}{ Folin-Ciocalteau } & \multirow{4}{*}{ Pakistan } & \multirow{4}{*}[67]{} \\
\hline & & $10,700 \mathrm{mgGAE} / 100 \mathrm{~g}$ & $10,700 \mathrm{mgGAE} / 100 \mathrm{~g}$ & & & & & \\
\hline & & $6160 \mathrm{mgGAE} / 100 \mathrm{~g}$ & $6160 \mathrm{mgGAE} / 100 \mathrm{~g}$ & & & & & \\
\hline & & $8210 \mathrm{mgGAE} / 100 \mathrm{~g}$ & $8210 \mathrm{mgGAE} / 100 \mathrm{~g}$ & & & & & \\
\hline & dried & $2070 \mathrm{mg}$ TAE$/ 100 \mathrm{~g}$ & $2070 \mathrm{mg}$ TAE$/ 100 \mathrm{~g}$ & Air-drying & Acetone/Water (7:3) & Folin-Ciocalteau & India & {$[68]$} \\
\hline & \multirow{2}{*}{ dried } & $1600 \mathrm{mgTEA} / 100 \mathrm{~g}^{\mathrm{d}}$ & $1600 \mathrm{mgTEA} / 100 \mathrm{~g}^{\mathrm{d}}$ & \multirow{2}{*}{ Air-drying } & \multirow{2}{*}{$80 \% \mathrm{EtOH}$} & \multirow{2}{*}{ Folin-Ciocalteau } & \multirow{2}{*}{ Nicaragua } & \multirow{2}{*}{ [69] } \\
\hline & & $3400 \mathrm{mgTEA} / 100 \mathrm{~g}^{\mathrm{e}}$ & $3400 \mathrm{mgTEA} / 100 \mathrm{~g}^{\mathrm{e}}$ & & & & & \\
\hline & \multirow{6}{*}{ dried } & $5350 \mathrm{mgCAE} / 100 \mathrm{~g}$ & $5350 \mathrm{mgCAE} / 100 \mathrm{~g}$ & \multirow{6}{*}{$\begin{array}{l}\text { Oven-drying } \\
\text { at } 60^{\circ} \mathrm{C} \text { for } 24 \mathrm{~h}\end{array}$} & Maceration with $70 \% \mathrm{EtOH}$ & \multirow{6}{*}{ Folin-Ciocalteau } & \multirow{6}{*}{ Thailand } & \multirow{6}{*}[70]{} \\
\hline & & $2930 \mathrm{mgCAE} / 100 \mathrm{~g}$ & $2930 \mathrm{mgCAE} / 100 \mathrm{~g}$ & & Maceration with $50 \%$ EtOH & & & \\
\hline & & $3710 \mathrm{mgCAE} / 100 \mathrm{~g}$ & $3710 \mathrm{mgCAE} / 100 \mathrm{~g}$ & & Percolation with $70 \%$ EtOH & & & \\
\hline & & $3280 \mathrm{mgCAE} / 100 \mathrm{~g}$ & $3280 \mathrm{mgCAE} / 100 \mathrm{~g}$ & & Percolation with $50 \%$ EtOH & & & \\
\hline & & $4550 \mathrm{mgCAE} / 100 \mathrm{~g}$ & $4550 \mathrm{mgCAE} / 100 \mathrm{~g}$ & & Soxhlet extraction with $70 \%$ EtOH & & & \\
\hline & & $4460 \mathrm{mgCAE} / 100 \mathrm{~g}$ & $4460 \mathrm{mgCAE} / 100 \mathrm{~g}$ & & Soxhlet extraction with $50 \%$ EtOH & & & \\
\hline & freeze-dried & $1535.6 \mathrm{mgGAE} / 100 \mathrm{~g}$ & $1535.6 \mathrm{mgGAE} / 100 \mathrm{~g}$ & Freeze-drying & $80 \% \mathrm{EtOH}$ & Folin-Ciocalteau & Florida, USA & [57] \\
\hline
\end{tabular}

Abbreviations: ${ }^{a}$ Mature/old leaves; ${ }^{b}$ Tender/young leaves; ${ }^{\mathrm{c}}$ Mean value of samples collected in different seasons; ${ }^{\mathrm{d}}$ Extracted leaves; ${ }^{\mathrm{e}}$ Unextracted leaves; N/A = Not available; $\mathrm{GAE}=$ Gallic acid equivalent; $\mathrm{TAE}=$ Tannin acid equivalent; $\mathrm{CAE}=$ Chlorogenic acid equivalent. 
Table 4. Phenolic acids content in Moringa oleifera leaves.

\begin{tabular}{|c|c|c|c|c|c|c|c|c|}
\hline Bioactive Compound & Leaves & $\begin{array}{l}\text { Value Found } \\
\text { in Literature }\end{array}$ & $\begin{array}{c}\text { Value Express as } \\
\text { Dry Weight } \\
\end{array}$ & $\begin{array}{l}\text { Drying } \\
\text { Method }\end{array}$ & Extractive Method & Analytical Method & Country & Reference \\
\hline \multicolumn{9}{|l|}{ Phenolic acids } \\
\hline \multirow{3}{*}{ Caffeic acid } & dried & ND & ND & N/A & $50 \% \mathrm{MeOH}$ & HPLC and MS/MS & India & [64] \\
\hline & dried & $0.409 \mathrm{mg} / \mathrm{g}$ & $0.409 \mathrm{mg} / \mathrm{g}$ & $\mathrm{N} / \mathrm{A}$ & $50 \% \mathrm{MeOH}, 100 \% \mathrm{MeOH}$ and water & HPLC & India & [65] \\
\hline & freeze-dried & $0.536 \mathrm{mg} / \mathrm{g}$ & $0.536 \mathrm{mg} / \mathrm{g}$ & Freeze-drying & $80 \% \mathrm{EtOH}$ & HPLC & Florida, USA & [57] \\
\hline \multirow{2}{*}{ Chlorogenic acid } & dried & $0.018 \mathrm{mg} / \mathrm{g}$ & $0.018 \mathrm{mg} / \mathrm{g}$ & N/A & $50 \% \mathrm{MeOH}$ & HPLC and MS/MS & India & [64] \\
\hline & dried & $0.489 \mathrm{mg} / \mathrm{g}$ & $0.489 \mathrm{mg} / \mathrm{g}$ & $\mathrm{N} / \mathrm{A}$ & Water at $80^{\circ} \mathrm{C}$ for $2 \mathrm{~h}$ & HPLC and MS/MS & India & [66] \\
\hline$o$-Coumaric acid & freeze-dried & $6.457 \mathrm{mg} / \mathrm{g}$ & $6.457 \mathrm{mg} / \mathrm{g}$ & Freeze-drying & $80 \% \mathrm{EtOH}$ & HPLC & Florida, USA & [57] \\
\hline$p$-Coumaric acid & freeze-dried & ND & ND & Freeze-drying & $80 \% \mathrm{EtOH}$ & HPLC & Florida, USA & [57] \\
\hline \multirow{3}{*}{ Ellagic acid } & dried & ND & ND & $\mathrm{N} / \mathrm{A}$ & $50 \% \mathrm{MeOH}, 100 \% \mathrm{MeOH}$ and water & HPLC & India & [65] \\
\hline & dried & $0.009 \mathrm{mg} / \mathrm{g}$ & $0.018 \mathrm{mg} / \mathrm{g}$ & $\mathrm{N} / \mathrm{A}$ & $50 \% \mathrm{MeOH}$ & HPLC and MS/MS & India & [64] \\
\hline & dried & $0.189 \mathrm{mg} / \mathrm{g}$ & $0.189 \mathrm{mg} / \mathrm{g}$ & N/A & Water at $80^{\circ} \mathrm{C}$ for $2 \mathrm{~h}$ & HPLC and MS/MS & India & [66] \\
\hline \multirow{3}{*}{ Ferulic acid } & dried & $0.078 \mathrm{mg} / \mathrm{g}$ & $0.078 \mathrm{mg} / \mathrm{g}$ & $\mathrm{N} / \mathrm{A}$ & $50 \% \mathrm{MeOH}$ & HPLC and MS/MS & India & [64] \\
\hline & dried & $0.078 \mathrm{mg} / \mathrm{g}$ & $0.078 \mathrm{mg} / \mathrm{g}$ & N/A & $50 \% \mathrm{MeOH}, 100 \% \mathrm{MeOH}$ and water & HPLC & India & [65] \\
\hline & dried & $0.128 \mathrm{mg} / \mathrm{g}$ & $0.128 \mathrm{mg} / \mathrm{g}$ & N/A & Water at $80^{\circ} \mathrm{C}$ for $2 \mathrm{~h}$ & HPLC and MS/MS & India & [66] \\
\hline \multirow{3}{*}{ Gallic acid } & dried & ND & ND & $\mathrm{N} / \mathrm{A}$ & $50 \% \mathrm{MeOH}, 100 \% \mathrm{MeOH}$ and water & HPLC & India & [65] \\
\hline & dried & $1.034 \mathrm{mg} / \mathrm{g}$ & $1.034 \mathrm{mg} / \mathrm{g}$ & N/A & $50 \% \mathrm{MeOH}$ & HPLC and MS/MS & India & [64] \\
\hline & dried & $1.034 \mathrm{mg} / \mathrm{g}$ & $1.034 \mathrm{mg} / \mathrm{g}$ & $\mathrm{N} / \mathrm{A}$ & Water at $80^{\circ} \mathrm{C}$ for $2 \mathrm{~h}$ & HPLC and MS/MS & India & [66] \\
\hline Gentistic acid & freeze-dried & ND & ND & Freeze-drying & $80 \% \mathrm{EtOH}$ & HPLC & Florida, USA & [57] \\
\hline Sinapic acid & freeze-dried & ND & ND & Freeze-drying & $80 \% \mathrm{EtOH}$ & HPLC & Florida, USA & [57] \\
\hline Syringic acid & freeze-dried & ND & ND & Freeze-drying & $80 \% \mathrm{EtOH}$ & HPLC & Florida, USA & [57] \\
\hline
\end{tabular}


Table 5. Flavonoids content in Moringa oleifera leaves.

\begin{tabular}{|c|c|c|c|c|c|c|c|c|}
\hline $\begin{array}{l}\text { Bioactive } \\
\text { Compound }\end{array}$ & Leaves & $\begin{array}{l}\text { Value Found } \\
\text { in Literature }\end{array}$ & $\begin{array}{c}\text { Value Express as } \\
\text { Dry Weight }\end{array}$ & Drying Method & Extractive Method & Analytical Method & Country & Reference \\
\hline \multicolumn{9}{|l|}{ Flavonoids } \\
\hline \multirow{26}{*}{$\begin{array}{c}\text { Total } \\
\text { flavonoids }\end{array}$} & dried & $1.29 \mathrm{mg} / \mathrm{g} \mathrm{a}^{\mathrm{a}}$ & $5.059 \mathrm{mg} / \mathrm{g}^{\text {a }}$ & Vacuum-drying & $\mathrm{MeOH}+\mathrm{HCl}+$ ascorbic acid & HPLC-DAD & Taiwan & [71] \\
\hline & & $6.0 \mathrm{mg} / \mathrm{g}^{\mathrm{a}, \mathrm{b}}$ & $6.0 \mathrm{mg} / \mathrm{g}^{\mathrm{a}, \mathrm{b}}$ & \multirow{3}{*}{ Air-drying } & \multirow{3}{*}{$70 \% \mathrm{MeOH}+0.1 \%$ acetic acid } & \multirow{3}{*}{$\mathrm{LC} / \mathrm{MS}$} & Ghana & \multirow{3}{*}{ [72] } \\
\hline & dried & $7.03 \mathrm{mg} / \mathrm{g}^{\mathrm{a}, \mathrm{b}}$ & $7.03 \mathrm{mg} / \mathrm{g}^{\mathrm{a}, \mathrm{b}}$ & & & & Senegal & \\
\hline & & $12.16 \mathrm{mg} / \mathrm{g}$ a,b & $12.16 \mathrm{mg} / \mathrm{g}$ a,b & & & & Zambia & \\
\hline & dried & $31.28 \mathrm{mgQE} / \mathrm{g}$ & $31.28 \mathrm{mgQE} / \mathrm{g}$ & $\mathrm{N} / \mathrm{A}$ & Water at $80^{\circ} \mathrm{C}$ for $2 \mathrm{~h}$ & HPLC and MS/MS & India & [66] \\
\hline & \multirow{2}{*}{ dried } & $27.0 \mathrm{mgQE} / \mathrm{g}^{\mathrm{c}}$ & $27.0 \mathrm{mgQE} / \mathrm{g}^{\mathrm{c}}$ & \multirow{2}{*}{ Shade-drying } & \multirow{2}{*}{ Water Soxhlet extraction for $18-20 \mathrm{~h}$} & \multirow{2}{*}{ Colorimetric method } & \multirow{2}{*}{ India } & \multirow{2}{*}{ [63] } \\
\hline & & $15.0 \mathrm{mgQE} / \mathrm{g}^{\mathrm{d}}$ & $15.0 \mathrm{mgQE} / \mathrm{g}^{\mathrm{d}}$ & & & & & \\
\hline & dried & $96.12 \mathrm{mg} \mathrm{ECE} / 100 \mathrm{~g}^{\mathrm{e}}$ & $96.12 \mathrm{mg} \mathrm{ECE} / 100 \mathrm{~g}^{\mathrm{e}}$ & Air-drying & $80 \% \mathrm{MeOH}$ & Colorimetric method & Pakistan & [56] \\
\hline & \multirow{4}{*}{ dried } & $60.6 \mathrm{mgCE} / \mathrm{g}$ & $60.6 \mathrm{mgCE} / \mathrm{g}$ & \multirow{4}{*}{ Air-drying } & $100 \% \mathrm{MeOH}$ & \multirow{4}{*}{$\begin{array}{l}\text { Spectrophotometric } \\
\text { method }\end{array}$} & \multirow{4}{*}{ Pakistan } & \multirow{4}{*}{ [67] } \\
\hline & & $86.6 \mathrm{mgCE} / \mathrm{g}$ & $86.6 \mathrm{mgCE} / \mathrm{g}$ & & $80 \% \mathrm{MeOH}$ & & & \\
\hline & & $53.3 \mathrm{mgCE} / \mathrm{g}$ & $53.3 \mathrm{mgCE} / \mathrm{g}$ & & $100 \% \mathrm{EtOH}$ & & & \\
\hline & & $62.1 \mathrm{mgCE} / \mathrm{g}$ & $62.1 \mathrm{mgCE} / \mathrm{g}$ & & $80 \% \mathrm{EtOH}$ & & & \\
\hline & \multirow{4}{*}{ dried } & $59.0 \mathrm{mgCE} / \mathrm{g}$ & $59.0 \mathrm{mgCE} / \mathrm{g}$ & \multirow{4}{*}{ Air-drying } & \multirow{4}{*}{ Extraction by reflux } & \multirow{4}{*}{$\begin{array}{l}\text { Spectrophotometric } \\
\text { method }\end{array}$} & \multirow{4}{*}{ Pakistan } & \multirow{4}{*}{ [67] } \\
\hline & & $72.9 \mathrm{mgCE} / \mathrm{g}$ & $72.9 \mathrm{mgCE} / \mathrm{g}$ & & & & & \\
\hline & & $41.9 \mathrm{mgCE} / \mathrm{g}$ & $41.9 \mathrm{mgCE} / \mathrm{g}$ & & & & & \\
\hline & & $53.1 \mathrm{mgCE} / \mathrm{g}$ & $53.1 \mathrm{mgCE} / \mathrm{g}$ & & & & & \\
\hline & \multirow{6}{*}{ dried } & $25.1 \mathrm{mgIQE} / \mathrm{g}$ & $25.1 \mathrm{mgIQE} / \mathrm{g}$ & \multirow{6}{*}{$\begin{array}{l}\text { Oven-drying at } \\
60^{\circ} \mathrm{C} \text { for } 24 \mathrm{~h}\end{array}$} & Maceration with $70 \%$ EtOH & \multirow{6}{*}{ Colorimetric method } & \multirow{6}{*}{ Thailand } & \multirow{6}{*}{ [70] } \\
\hline & & $12.3 \mathrm{mgIQE} / \mathrm{g}$ & $12.3 \mathrm{mgIQE} / \mathrm{g}$ & & Maceration with $50 \%$ EtOH & & & \\
\hline & & $18.0 \mathrm{mgIQE} / \mathrm{g}$ & $18.0 \mathrm{mgIQE} / \mathrm{g}$ & & Percolation with $70 \%$ EtOH & & & \\
\hline & & $14.6 \mathrm{mgIQE} / \mathrm{g}$ & $14.6 \mathrm{mgIQE} / \mathrm{g}$ & & Percolation with $50 \%$ EtOH & & & \\
\hline & & $24.5 \mathrm{mgIQE} / \mathrm{g}$ & $24.5 \mathrm{mgIQE} / \mathrm{g}$ & & Soxhlet extraction with $70 \% \mathrm{EtOH}$ & & & \\
\hline & & $12.7 \mathrm{mgIQE} / \mathrm{g}$ & $12.7 \mathrm{mgIQE} / \mathrm{g}$ & & Soxhlet extraction with $50 \%$ EtOH & & & \\
\hline & freeze-dried & $61.62 \mathrm{mgRE} / \mathrm{g}$ & $61.62 \mathrm{mgRE} / \mathrm{g}$ & Freeze-drying & $80 \% \mathrm{EtOH}$ & $\begin{array}{c}\text { Spectrophotometric } \\
\text { method }\end{array}$ & Florida, USA & [57] \\
\hline & \multirow{3}{*}{ freeze-dried } & $44.3 \mathrm{mgRE} / \mathrm{g}$ & $44.3 \mathrm{mgRE} / \mathrm{g}$ & & & & Nicaragua & \\
\hline & & $21.0 \mathrm{mgRE} / \mathrm{g}$ & $21.0 \mathrm{mgRE} / \mathrm{g}$ & Freeze-drying & $80 \% \mathrm{MeOH}$ & Spectrophotometric & India & [58] \\
\hline & & $38.1 \mathrm{mgRE} / \mathrm{g}$ & $38.1 \mathrm{mgRE} / \mathrm{g}$ & & & method & Niger & \\
\hline
\end{tabular}


Table 5. Cont.

\begin{tabular}{|c|c|c|c|c|c|c|c|c|}
\hline $\begin{array}{c}\text { Bioactive } \\
\text { Compound }\end{array}$ & Leaves & $\begin{array}{l}\text { Value Found } \\
\text { in Literature }\end{array}$ & $\begin{array}{c}\text { Value Express as } \\
\text { Dry Weight }\end{array}$ & Drying Method & Extractive Method & Analytical Method & Country & Reference \\
\hline Apigenin & dried & ND & ND & $\mathrm{N} / \mathrm{A}$ & $\mathrm{MeOH}+\mathrm{HCl}+$ ascorbic acid & HPLC & Taiwan & [71] \\
\hline Daidzein & dried & ND & ND & N/A & $50 \% \mathrm{MeOH}, 100 \% \mathrm{MeOH}$ and water & HPLC & India & [65] \\
\hline Epicatechin & freeze-dried & $5.68 \mathrm{mg} / \mathrm{g}$ & $5.68 \mathrm{mg} / \mathrm{g}$ & Freeze-drying & $80 \% \mathrm{EtOH}$ & HPLC & Florida, USA & [57] \\
\hline Genistein & dried & ND & ND & $\mathrm{N} / \mathrm{A}$ & $50 \% \mathrm{MeOH}, 100 \% \mathrm{MeOH}$ and water & HPLC & India & [65] \\
\hline \multirow[b]{2}{*}{ Isorhamnetin } & dried & $0.03 \mathrm{mg} / \mathrm{g}$ & $0.118 \mathrm{mg} / \mathrm{g}$ & Vacuum-drying & $\mathrm{MeOH}+\mathrm{HCl}+$ ascorbic acid & HPLC & Taiwan & [71] \\
\hline & freeze-dried & $\begin{array}{l}0.13 \mathrm{mg} / \mathrm{g}^{\mathrm{f}} \\
0.18 \mathrm{mg} / \mathrm{g}^{\mathrm{h}}\end{array}$ & $\begin{array}{l}0.52 \mathrm{mg} / \mathrm{g}^{\mathrm{f}, \mathrm{g}} \\
0.72 \mathrm{mg} / \mathrm{g}^{\mathrm{g}, \mathrm{h}}\end{array}$ & Freeze-drying & $70 \% \mathrm{MeOH}$ & $\begin{array}{c}\text { HPLC-DAD-electrospray } \\
\text { mass spectrometry }\end{array}$ & Ghana & [73] \\
\hline \multirow{17}{*}{ Kaempferol } & dried & $0.04 \mathrm{mg} / \mathrm{g}$ & $0.04 \mathrm{mg} / \mathrm{g}$ & Air-drying & $\mathrm{MeOH}+1 \% v / v \mathrm{HCl}+\mathrm{TBHQ}$ & HPLC & Pakistan & [74] \\
\hline & dried & ND & ND & N/A & $50 \% \mathrm{MeOH}$ & HPLC and MS/MS & India & [64] \\
\hline & dried & $2.360 \mathrm{mg} / \mathrm{g}$ & $2.360 \mathrm{mg} / \mathrm{g}$ & $\mathrm{N} / \mathrm{A}$ & $50 \% \mathrm{MeOH}, 100 \% \mathrm{MeOH}$ and water & HPLC & India & [65] \\
\hline & dried & $0.198 \mathrm{mg} / \mathrm{g}$ & $0.198 \mathrm{mg} / \mathrm{g}$ & $\mathrm{N} / \mathrm{A}$ & Water at $80^{\circ} \mathrm{C}$ for $2 \mathrm{~h}$ & HPLC and MS/MS & India & [66] \\
\hline & dried & $0.36 \mathrm{mg} / \mathrm{g}$ & $1.412 \mathrm{mg} / \mathrm{g}$ & Vacuum-drying & $\mathrm{MeOH}+\mathrm{HCl}+10 \mathrm{mg}$ ascorbic acid & HPLC & Taiwan & [71] \\
\hline & \multirow{3}{*}{ dried } & $0.8 \mathrm{mg} / \mathrm{g}$ & $0.8 \mathrm{mg} / \mathrm{g}$ & \multirow{3}{*}{ Air-drying } & \multirow{3}{*}{$70 \% \mathrm{MeOH}+0.1 \%$ acetic acid } & \multirow{3}{*}{$\mathrm{LC} / \mathrm{MS}$} & Ghana & \multirow{3}{*}{ [72] } \\
\hline & & $1.23 \mathrm{mg} / \mathrm{g}$ & $1.23 \mathrm{mg} / \mathrm{g}$ & & & & Senegal & \\
\hline & & $4.59 \mathrm{mg} / \mathrm{g}$ & $4.59 \mathrm{mg} / \mathrm{g}$ & & & & Zambia & \\
\hline & freeze-dried & $\begin{array}{l}0.98 \mathrm{mg} / \mathrm{g}^{\mathrm{f}} \\
0.54 \mathrm{mg} / \mathrm{g}^{\mathrm{h}}\end{array}$ & $\begin{array}{l}3.92 \mathrm{mg} / \mathrm{g}^{\mathrm{f}, \mathrm{g}} \\
2.16 \mathrm{mg} / \mathrm{g}^{\mathrm{g}, \mathrm{h}}\end{array}$ & Freeze-drying & $70 \% \mathrm{MeOH}$ & $\begin{array}{c}\text { HPLC-DAD-electrospray } \\
\text { mass spectrometry }\end{array}$ & Ghana & [73] \\
\hline & \multirow{3}{*}{ freeze-dried } & $2.25 \mathrm{mg} / \mathrm{g}$ & $2.25 \mathrm{mg} / \mathrm{g}$ & \multirow{3}{*}{ Freeze-drying } & \multirow{3}{*}{$80 \% \mathrm{MeOH}$} & \multirow{3}{*}{ HPLC-DAD } & Nicaragua & \multirow{3}{*}[58]{} \\
\hline & & $1.75 \mathrm{mg} / \mathrm{g}$ & $1.75 \mathrm{mg} / \mathrm{g}$ & & & & India & \\
\hline & & $1.05 \mathrm{mg} / \mathrm{g}$ & $1.05 \mathrm{mg} / \mathrm{g}$ & & & & Niger & \\
\hline & \multirow{5}{*}{ freeze-dried } & $2.9 \mathrm{mg} / \mathrm{g}^{\mathrm{d}}$ & $2.9 \mathrm{mg} / \mathrm{g}^{\mathrm{d}}$ & \multirow{5}{*}{ Freeze-drying } & \multirow{5}{*}{$70 \% \mathrm{MeOH}$} & \multirow{5}{*}{ LC/MS } & Malawi & \multirow{5}{*}{ [75] } \\
\hline & & $2.3 \mathrm{mg} / \mathrm{g}$ & $2.3 \mathrm{mg} / \mathrm{g}$ & & & & Senegal & \\
\hline & & $3.5 \mathrm{mg} / \mathrm{g}$ & $3.5 \mathrm{mg} / \mathrm{g}$ & & & & Nicaragua & \\
\hline & & $0.3 \mathrm{mg} / \mathrm{g}^{\mathrm{c}}$ & $0.3 \mathrm{mg} / \mathrm{g}^{\mathrm{c}}$ & & & & ECHO & \\
\hline & & $0.16 \mathrm{mg} / \mathrm{g}^{\mathrm{d}}$ & $0.16 \mathrm{mg} / \mathrm{g}^{\mathrm{d}}$ & & & & ECHO & \\
\hline Luteolin & dried & ND & ND & N/A & $\mathrm{MeOH}+\mathrm{HCl}+$ ascorbic acid & HPLC & Taiwan & [71] \\
\hline
\end{tabular}


Table 5. Cont.

\begin{tabular}{|c|c|c|c|c|c|c|c|c|}
\hline $\begin{array}{c}\text { Bioactive } \\
\text { Compound }\end{array}$ & Leaves & $\begin{array}{l}\text { Value Found } \\
\text { in Literature }\end{array}$ & $\begin{array}{c}\text { Value Express as } \\
\text { Dry Weight }\end{array}$ & Drying Method & Extractive Method & Analytical Method & Country & Reference \\
\hline Myricetin & dried & $5.804 \mathrm{mg} / \mathrm{g}$ & $5.804 \mathrm{mg} / \mathrm{g}$ & Air-drying & $\mathrm{MeOH}+1 \% v / v \mathrm{HCl}+\mathrm{TBHQ}$ & HPLC & Pakistan & [74] \\
\hline \multirow{18}{*}{ Quercetin } & dried & $0.281 \mathrm{mg} / \mathrm{g}$ & $0.281 \mathrm{mg} / \mathrm{g}$ & Air-drying & $\mathrm{MeOH}+1 \% v / v \mathrm{HCl}+\mathrm{TBHQ}$ & HPLC & Pakistan & [74] \\
\hline & dried & $0.207 \mathrm{mg} / \mathrm{g}$ & $0.207 \mathrm{mg} / \mathrm{g}$ & $\mathrm{N} / \mathrm{A}$ & $50 \% \mathrm{MeOH}$ & HPLC and MS/MS & India & [64] \\
\hline & dried & $0.207 \mathrm{mg} / \mathrm{g}$ & $0.207 \mathrm{mg} / \mathrm{g}$ & $\mathrm{N} / \mathrm{A}$ & $50 \% \mathrm{MeOH}, 100 \% \mathrm{MeOH}$ and water & HPLC & India & [65] \\
\hline & dried & $0.807 \mathrm{mg} / \mathrm{g}$ & $0.807 \mathrm{mg} / \mathrm{g}$ & $\mathrm{N} / \mathrm{A}$ & Water at $80^{\circ} \mathrm{C}$ for $2 \mathrm{~h}$ & HPLC and MS/MS & India & [66] \\
\hline & dried & $0.90 \mathrm{mg} / \mathrm{g}$ & $3.529 \mathrm{mg} / \mathrm{g}$ & Vacuum-drying & $\mathrm{MeOH}+\mathrm{HCl}+10 \mathrm{mg}$ ascorbic acid & HPLC & Taiwan & [71] \\
\hline & \multirow{3}{*}{ dried } & $5.2 \mathrm{mg} / \mathrm{g}$ & $5.2 \mathrm{mg} / \mathrm{g}$ & \multirow{3}{*}{ Air-drying } & \multirow{3}{*}{$70 \% \mathrm{MeOH}+0.1 \%$ acetic acid } & \multirow{3}{*}{$\mathrm{LC} / \mathrm{MS}$} & Ghana & \multirow{3}{*}{ [72] } \\
\hline & & $5.8 \mathrm{mg} / \mathrm{g}$ & $5.8 \mathrm{mg} / \mathrm{g}$ & & & & Senegal & \\
\hline & & $7.57 \mathrm{mg} / \mathrm{g}$ & $7.57 \mathrm{mg} / \mathrm{g}$ & & & & Zambia & \\
\hline & \multirow{2}{*}{ freeze-dried } & $3.21 \mathrm{mg} / \mathrm{g}^{\mathrm{f}}$ & $12.84 \mathrm{mg} / \mathrm{g}, \mathrm{g}$ & \multirow{2}{*}{ Freeze-drying } & \multirow{2}{*}{$70 \% \mathrm{MeOH}$} & HPLC-DAD-electrospray & \multirow{2}{*}{ Ghana } & \multirow{2}{*}{ [73] } \\
\hline & & $4.16 \mathrm{mg} / \mathrm{g}^{\mathrm{h}}$ & $16.64 \mathrm{mg} / \mathrm{g}^{\mathrm{g}, \mathrm{h}}$ & & & mass spectrometry & & \\
\hline & \multirow{3}{*}{ freeze-dried } & $9.26 \mathrm{mg} / \mathrm{g}$ & $9.26 \mathrm{mg} / \mathrm{g}$ & \multirow{3}{*}{ Freeze-drying } & \multirow{3}{*}{$80 \% \mathrm{MeOH}$} & \multirow{3}{*}{ HPLC-DAD } & Nicaragua & \multirow{3}{*}[58]{} \\
\hline & & $6.34 \mathrm{mg} / \mathrm{g}$ & $6.34 \mathrm{mg} / \mathrm{g}$ & & & & India & \\
\hline & & $7.70 \mathrm{mg} / \mathrm{g}$ & $7.70 \mathrm{mg} / \mathrm{g}$ & & & & Niger & \\
\hline & \multirow{5}{*}{ freeze-dried } & $5.47 \mathrm{mg} / \mathrm{g}^{\mathrm{b}}$ & $5.47 \mathrm{mg} / \mathrm{g}^{\mathrm{b}}$ & \multirow{5}{*}{ Freeze-drying } & \multirow{5}{*}{$70 \% \mathrm{MeOH}$} & \multirow{5}{*}{$\mathrm{LC} / \mathrm{MS}$} & Malawi & \multirow{5}{*}[75]{} \\
\hline & & $9.1 \mathrm{mg} / \mathrm{g}$ & $9.1 \mathrm{mg} / \mathrm{g}$ & & & & Senegal & \\
\hline & & $15.2 \mathrm{mg} / \mathrm{g}$ & $15.2 \mathrm{mg} / \mathrm{g}$ & & & & Nicaragua & \\
\hline & & $0.58 \mathrm{mg} / \mathrm{g}^{\mathrm{c}}$ & $0.58 \mathrm{mg} / \mathrm{g}^{\mathrm{c}}$ & & & & $\mathrm{ECHO}$ & \\
\hline & & $0.46 \mathrm{mg} / \mathrm{g}^{\mathrm{d}}$ & $0.46 \mathrm{mg} / \mathrm{g}^{\mathrm{d}}$ & & & & ECHO & \\
\hline \multirow{3}{*}{ Rutin } & dried & $0.390 \mathrm{mg} / \mathrm{g}$ & $0.390 \mathrm{mg} / \mathrm{g}$ & N/A & $50 \% \mathrm{MeOH}, 100 \% \mathrm{MeOH}$ and water & HPLC & India & [65] \\
\hline & dried & ND & ND & $\mathrm{N} / \mathrm{A}$ & $50 \% \mathrm{MeOH}$ & HPLC and MS/MS & India & [64] \\
\hline & freeze-dried & $1.674 \mathrm{mg} / \mathrm{g}$ & $1.674 \mathrm{mg} / \mathrm{g}$ & Freeze-drying & $80 \% \mathrm{EtOH}$ & HPLC & Florida, USA & [57] \\
\hline
\end{tabular}

Abbreviations: ${ }^{\mathrm{a}}$ Obtained from the sum of single flavonoids measured; ${ }^{\mathrm{b}}$ Mean value of different samples; ${ }^{\mathrm{c}}$ Mature/old leaves; ${ }^{\mathrm{d}}$ Tender/young leaves; ${ }^{\mathrm{e}}$ Mean value of samples collected in different seasons; ${ }^{\mathrm{f}}$ Vegetative plants; ${ }^{\mathrm{g}}$ Obtained considering a moisture of $75 \%$; ${ }^{\mathrm{h}}$ Flowering plants; ND = Not detected; N/A = Not available; $\mathrm{QE}=$ Quercetin equivalent; $\mathrm{ECE}=$ Epicatechine equivalent; $\mathrm{CE}=$ Catechin equivalent; $\mathrm{IQE}=$ Isoquercetin equivalent; $\mathrm{RE}=$ Rutein equivalent. 
Table 6. Glucosinolates content in Moringa oleifera leaves.

\begin{tabular}{|c|c|c|c|c|c|c|c|c|}
\hline Bioactive Compound & Leaves & Value Found in Literature & Value Express as Dry Weight & Drying Method & Extractive Method & Analytical Method & Country & Reference \\
\hline \multicolumn{9}{|l|}{ Glucosinolates } \\
\hline \multirow{2}{*}{ Benzyl } & freeze-dried & $\mathrm{ND}^{\mathrm{a}} \mathrm{ND}^{\mathrm{b}}$ & $\mathrm{ND}^{\mathrm{a}} \mathrm{ND}^{\mathrm{b}}$ & Freeze-drying & $70 \% \mathrm{MeOH}$ & $\begin{array}{c}\text { HPLC-DAD-electrospray } \\
\text { mass spectrometry }\end{array}$ & Ghana & [73] \\
\hline & freeze-dried & $\mathrm{ND}^{\mathrm{c}} \mathrm{ND}^{\mathrm{d}}$ & $\mathrm{ND}^{\mathrm{c}} \mathrm{ND}^{\mathrm{d}}$ & Freeze-drying & $70 \% \mathrm{MeOH}$ & LC/MS & $\begin{array}{c}\text { Many } \\
\text { countries }\end{array}$ & {$[75]$} \\
\hline 4-hydroxybenzyl (sinalbin) & freeze-dried & $0.59 \mathrm{mg} / \mathrm{g}^{\mathrm{a}} \mathrm{ND}^{\mathrm{b}}$ & $2.36 \mathrm{mg} / \mathrm{g}^{\mathrm{a}, \mathrm{e}} \mathrm{ND}^{\mathrm{b}, \mathrm{e}}$ & Freeze-drying & $70 \% \mathrm{MeOH}$ & $\begin{array}{c}\text { HPLC-DAD-electrospray } \\
\text { mass spectrometry }\end{array}$ & Ghana & [73] \\
\hline \multirow{2}{*}{$\begin{array}{l}\text { 4-( } \alpha \text {-L-rhamnopyranosyloxy)- } \\
\text { benzyl }\end{array}$} & freeze-dried & $\begin{array}{l}5.64 \mathrm{mg} / \mathrm{g}^{\mathrm{a}} \\
5.46 \mathrm{mg} / \mathrm{g}^{\mathrm{b}}\end{array}$ & $\begin{array}{l}22.56 \mathrm{mg} / \mathrm{g}^{\mathrm{a}, \mathrm{e}} \\
21.84 \mathrm{mg} / \mathrm{g}^{\mathrm{b}, \mathrm{e}}\end{array}$ & Freeze-drying & $70 \% \mathrm{MeOH}$ & $\begin{array}{c}\text { HPLC-DAD-electrospray } \\
\text { mass spectrometry }\end{array}$ & Ghana & [73] \\
\hline & freeze-dried & $\begin{array}{l}33.9 \mathrm{mg} / \mathrm{g}^{\mathrm{c}} \\
59.4 \mathrm{mg} / \mathrm{g}^{\mathrm{d}}\end{array}$ & $\begin{array}{l}33.9 \mathrm{mg} / \mathrm{g}^{\mathrm{c}} \\
59.4 \mathrm{mg} / \mathrm{g}^{\mathrm{d}}\end{array}$ & Freeze-drying & $70 \% \mathrm{MeOH}$ & LC/MS & $\begin{array}{c}\text { Many } \\
\text { countries }\end{array}$ & {$[75]$} \\
\hline \multirow{2}{*}{$\begin{array}{c}\text { 4-O- }(\alpha-\mathrm{L}- \\
\text { acetylrhamnopyranosyloxy)- } \\
\text { benzyl isomer } 1\end{array}$} & freeze-dried & $\begin{array}{l}0.69 \mathrm{mg} / \mathrm{g}^{\mathrm{a}} \\
0.54 \mathrm{mg} / \mathrm{g}^{\mathrm{b}}\end{array}$ & $\begin{array}{l}2.76 \mathrm{mg} / \mathrm{g}^{\mathrm{a}, \mathrm{e}} \\
2.16 \mathrm{mg} / \mathrm{g}^{\mathrm{b}, \mathrm{e}}\end{array}$ & Freeze-drying & $70 \% \mathrm{MeOH}$ & $\begin{array}{c}\text { HPLC-DAD-electrospray } \\
\text { mass spectrometry }\end{array}$ & Ghana & {$[73]$} \\
\hline & freeze-dried & $\begin{array}{l}2.9 \mathrm{mg} / \mathrm{g}^{\mathrm{c}} \\
5.0 \mathrm{mg} / \mathrm{g}^{\mathrm{d}}\end{array}$ & $\begin{array}{l}2.9 \mathrm{mg} / \mathrm{g}^{\mathrm{c}} \\
5.0 \mathrm{mg} / \mathrm{g}^{\mathrm{d}}\end{array}$ & Freeze-drying & $70 \% \mathrm{MeOH}$ & LC/MS & $\begin{array}{c}\text { Many } \\
\text { countries }\end{array}$ & [75] \\
\hline \multirow{2}{*}{$\begin{array}{l}\text { 4-O-( } \alpha-\mathrm{L}- \\
\text { acetylrhamnopyranosyloxy)- } \\
\text { benzyl isomer } 2\end{array}$} & freeze-dried & $\begin{array}{l}0.45 \mathrm{mg} / \mathrm{g}^{\mathrm{a}} \\
0.38 \mathrm{mg} / \mathrm{g}^{\mathrm{b}}\end{array}$ & $\begin{array}{l}1.80 \mathrm{mg} / \mathrm{g}^{\mathrm{a}, \mathrm{e}} \\
1.52 \mathrm{mg} / \mathrm{g}^{\mathrm{b}, \mathrm{e}}\end{array}$ & Freeze-drying & $70 \% \mathrm{MeOH}$ & $\begin{array}{c}\text { HPLC-DAD-electrospray } \\
\text { mass spectrometry }\end{array}$ & Ghana & {$[73]$} \\
\hline & freeze-dried & $\begin{array}{l}1.2 \mathrm{mg} / \mathrm{g}^{\mathrm{c}} \\
1.5 \mathrm{mg} / \mathrm{g}^{\mathrm{d}}\end{array}$ & $\begin{array}{l}1.2 \mathrm{mg} / \mathrm{g}^{\mathrm{c}} \\
1.5 \mathrm{mg} / \mathrm{g}^{\mathrm{d}}\end{array}$ & Freeze-drying & $70 \% \mathrm{MeOH}$ & $\mathrm{LC} / \mathrm{MS}$ & $\begin{array}{c}\text { Many } \\
\text { countries }\end{array}$ & {$[75]$} \\
\hline \multirow{2}{*}{$\begin{array}{c}\text { 4-O-( } \alpha-\mathrm{L}- \\
\text { acetylrhamnopyranosyloxy)- } \\
\text { benzyl isomer } 3\end{array}$} & freeze-dried & $\begin{array}{l}5.04 \mathrm{mg} / \mathrm{g}^{\mathrm{a}} \\
3.19 \mathrm{mg} / \mathrm{g} \mathrm{b}^{\mathrm{b}}\end{array}$ & $\begin{array}{l}20.16 \mathrm{mg} / \mathrm{g} \text { a,e } \\
12.76 \mathrm{mg} / \mathrm{g}^{\mathrm{b}, \mathrm{e}}\end{array}$ & Freeze-drying & $70 \% \mathrm{MeOH}$ & $\begin{array}{c}\text { HPLC-DAD-electrospray } \\
\text { mass spectrometry }\end{array}$ & Ghana & [73] \\
\hline & freeze-dried & $\begin{array}{l}17.4 \mathrm{mg} / \mathrm{g}^{\mathrm{c}} \\
50.2 \mathrm{mg} / \mathrm{g}^{\mathrm{d}}\end{array}$ & $\begin{array}{l}17.4 \mathrm{mg} / \mathrm{g}^{\mathrm{c}} \\
50.2 \mathrm{mg} / \mathrm{g}^{\mathrm{d}}\end{array}$ & Freeze-drying & $70 \% \mathrm{MeOH}$ & LC/MS & $\begin{array}{c}\text { Many } \\
\text { countries }\end{array}$ & [75] \\
\hline
\end{tabular}

Abbreviations: ${ }^{\mathrm{a}}$ Vegetative plants; ${ }^{\mathrm{b}}$ Flowering plants; ${ }^{\mathrm{c}}$ Mature/old leaves; ${ }^{\mathrm{d}}$ Tender/young leaves; ${ }^{\mathrm{e}}$ Obtained considering a moisture of $75 \% ; \mathrm{ND}=\mathrm{Not}$ detected. 
Table 7. Tannins content in Moringa oleifera leaves.

\begin{tabular}{|c|c|c|c|c|c|c|c|c|}
\hline $\begin{array}{c}\text { Bioactive } \\
\text { Compound }\end{array}$ & Leaves & $\begin{array}{l}\text { Value Found } \\
\text { in Literature }\end{array}$ & $\begin{array}{c}\text { Value Express as } \\
\text { Dry Weight } \\
\end{array}$ & Drying Method & Extractive Method & Analytical Method & Country & Reference \\
\hline \multicolumn{9}{|l|}{ Tannins } \\
\hline \multirow{5}{*}{ Total tannins } & dried & $13.2 \mathrm{gTAE} / \mathrm{kg}$ & $13.2 \mathrm{gTAE} / \mathrm{kg}$ & Air-drying & Acetone/Water $(7: 3)$ & Folin-Ciocalteau modified & India & [68] \\
\hline & dried & $\mathrm{ND}^{\mathrm{a}} 14.0 \mathrm{gTAE} / \mathrm{kg}^{\mathrm{b}}$ & $\mathrm{ND}^{\mathrm{a}} 14.0 \mathrm{gTAE} / \mathrm{kg}^{\mathrm{b}}$ & Air-drying & $80 \% \mathrm{EtOH}$ & Folin-Ciocalteau modified & Nicaragua & [69] \\
\hline & dried & $20.6 \mathrm{~g} / \mathrm{kg}$ & $20.6 \mathrm{~g} / \mathrm{kg}$ & Air-drying at $35^{\circ} \mathrm{C}$ for $24 \mathrm{~h}$ & Double lipid extraction with n-hexane (1:5) & N/A & Brazil & [76] \\
\hline & freeze-dried & $12 \mathrm{~g} / \mathrm{kg}$ & $12 \mathrm{~g} / \mathrm{kg}$ & Freeze-drying & $80 \% \mathrm{MeOH}$ & Folin-Ciocalteau modified & Nicaragua & [77] \\
\hline & freeze-dried & $5 \mathrm{~g} / \mathrm{kg}$ & $5 \mathrm{~g} / \mathrm{kg}$ & Freeze-drying & $80 \% \mathrm{EtOH}$ & Folin-Ciocalteau modified & Niger & [78] \\
\hline \multirow{2}{*}{ Condensed tannins } & dried & $1.05 \mathrm{gLE} / \mathrm{kg}$ & $1.05 \mathrm{gLE} / \mathrm{kg}$ & Air-drying & Acetone/Water (7:3) & Butanol-HCl-iron method & India & {$[68]$} \\
\hline & dried & $3.12 \mathrm{~g} / \mathrm{kg}$ & $3.12 \mathrm{~g} / \mathrm{kg}$ & Air-dried under shade & N/A & Butanol-HCl-iron method & South Africa & [61] \\
\hline
\end{tabular}

Abbreviations: ${ }^{\text {a }}$ Extracted leaves; ${ }^{\mathrm{b}}$ Unextracted leaves; TAE $=$ Tannin acid equivalent; LE $=$ Leucocyanidin equivalent; ND = Not detected; N/A = Not available.

Table 8. Saponins content in Moringa oleifera leaves.

\begin{tabular}{|c|c|c|c|c|c|c|c|c|}
\hline $\begin{array}{c}\text { Bioactive } \\
\text { Compound }\end{array}$ & Leaves & $\begin{array}{l}\text { Value Found } \\
\text { in Literature }\end{array}$ & $\begin{array}{c}\text { Value Express as } \\
\text { Dry Weight }\end{array}$ & Drying Method & Extractive Method & Analytical Method & Country & Reference \\
\hline \multicolumn{9}{|l|}{ Saponins } \\
\hline \multirow{3}{*}{ Total saponins } & dried & $\begin{array}{c}2.0 \mathrm{gDE} / \mathrm{kg}^{\mathrm{a}} \\
50.0 \mathrm{gDE} / \mathrm{kg}^{\mathrm{b}}\end{array}$ & $\begin{array}{c}2.0 \mathrm{gDE} / \mathrm{kg}^{\mathrm{a}} \\
50.0 \mathrm{gDE} / \mathrm{kg}^{\mathrm{b}}\end{array}$ & Air-drying & $80 \% \mathrm{EtOH}$ & Spectrophotometric method & Nicaragua & {$[69]$} \\
\hline & freeze-dried & $81 \mathrm{gDE} / \mathrm{kg}$ & $81 \mathrm{gDE} / \mathrm{kg}$ & Freeze-drying & $80 \% \mathrm{MeOH}$ & Spectrophotometric method & Nicaragua & [77] \\
\hline & freeze-dried & $64 \mathrm{gDE} / \mathrm{kg}$ & $64 \mathrm{gDE} / \mathrm{kg}$ & Freeze-drying & $80 \% \mathrm{EtOH}$ & Spectrophotometric method & Niger & [78] \\
\hline
\end{tabular}

Abbreviations: ${ }^{\text {a }}$ Extracted leaves; ${ }^{\mathrm{b}}$ Unextracted leaves; DE = Diosgenin equivalent. 
Table 9. Oxalates and phytates content in Moringa oleifera leaves.

\begin{tabular}{|c|c|c|c|c|c|c|c|c|}
\hline Bioactive Compound & Leaves & $\begin{array}{l}\text { Value Found } \\
\text { in Literature }\end{array}$ & $\begin{array}{c}\text { Value Express as } \\
\text { Dry Weight }\end{array}$ & Drying Method & Extractive Method & Analytical Method & Country & Reference \\
\hline \multicolumn{9}{|l|}{ Oxalates and phytates } \\
\hline \multirow{4}{*}{ Oxalates } & & $430 \mathrm{mg} / 100 \mathrm{~g}$ & $430 \mathrm{mg} / 100 \mathrm{~g}$ & Sun-drying for 4 days & \multirow{3}{*}{ N/A } & \multirow{3}{*}{ AOAC 2004} & \multirow{3}{*}{ India } & \multirow{3}{*}[55]{} \\
\hline & dried & $500 \mathrm{mg} / 100 \mathrm{~g}$ & $500 \mathrm{mg} / 100 \mathrm{~g}$ & Shadow-drying for 6 days & & & & \\
\hline & & $450 \mathrm{mg} / 100 \mathrm{~g}$ & $450 \mathrm{mg} / 100 \mathrm{~g}$ & Oven-drying at $60^{\circ} \mathrm{C}$ for $1 \mathrm{~h}$ & & & & \\
\hline & dried & $1050 \mathrm{mg} / 100 \mathrm{~g}$ & $1050 \mathrm{mg} / 100 \mathrm{~g}$ & Air-drying at $35^{\circ} \mathrm{C}$ for $24 \mathrm{~h}$ & $\begin{array}{c}\text { Double lipid extraction } \\
\text { with n-hexane (1:5) }\end{array}$ & $\mathrm{N} / \mathrm{A}$ & Brazil & {$[76]$} \\
\hline \multirow{3}{*}{ Phytates } & dried & $\begin{array}{l}25.0 \mathrm{~g} / \mathrm{kg}^{\mathrm{a}} \\
31.0 \mathrm{~g} / \mathrm{kg}^{\mathrm{b}}\end{array}$ & $\begin{array}{l}25.0 \mathrm{~g} / \mathrm{kg}^{\mathrm{a}} \\
31.0 \mathrm{~g} / \mathrm{kg}^{\mathrm{b}}\end{array}$ & Air-drying & $3.5 \% \mathrm{HCl}$ for $1 \mathrm{~h}$ & Colorimetric method & Nicaragua & [69] \\
\hline & freeze-dried & $21.0 \mathrm{~g} / \mathrm{kg}$ & $21.0 \mathrm{~g} / \mathrm{kg}$ & Freeze-drying & $3.5 \% \mathrm{HCl}$ for $1 \mathrm{~h}$ & Colorimetric method & Nicaragua & [77] \\
\hline & freeze-dried & $23.0 \mathrm{~g} / \mathrm{kg}$ & $23.0 \mathrm{~g} / \mathrm{kg}$ & Freeze-drying & $3.5 \% \mathrm{HCl}$ for $1 \mathrm{~h}$ & Colorimetric method & Niger & [78] \\
\hline
\end{tabular}

Abbreviations: ${ }^{a}$ Extracted leaves; ${ }^{b}$ Unextracted leaves; N/A $=$ Not available. 


\subsection{Vitamins}

Fresh leaves of Moringa oleifera are reported to contain 11,300-23,000 IU of vitamin A [14,52]. Vitamin A plays key roles in many physiological processes such as vision, reproduction, embryonic growth and development, immune competence, cell differentiation, cell proliferation and apoptosis, maintenance of epithelial tissue, and brain function. Its deficiency is still prevalent in many developing countries, and considered responsible for child and maternal mortality [79].

Fresh leaves of Moringa oleifara are also a good source of carotenoids with pro-vitamin A action. They contain $6.6-6.8 \mathrm{mg} / 100 \mathrm{~g}[53,62]$ of $\beta$-carotene, greater that carrots, pumpkin and apricots $(6.9,3.6$ and $2.2 \mathrm{mg} / 100 \mathrm{~g}$, respectively) [80].

$\beta$-carotene is more concentrated in the dried leaves, with amounts ranging from 17.6 to $39.6 \mathrm{mg} / 100 \mathrm{~g}$ of dry weight (DW) $[53,55,61]$. This wide range may be explained by the different environmental conditions existing among different origin countries, genetic of the plant, drying method [55] and the different extraction and analysis methods employed as well. Freeze-drying seems to be the most conservative dehydration method. In freeze-drying leaves the $\beta$-carotene content is approximately $66 \mathrm{mg} / 100 \mathrm{~g}$ [57].

Moringa oleifera is an interesting source of vitamin C. Fresh leaves contain approximately $200 \mathrm{mg} / 100 \mathrm{~g}$ [14], greater than orange [80]. These amounts are of particular interest, as the vitamin C intervenes in the synthesis and metabolism of many compounds, like tyrosine, folic acid and tryptophan, hydroxylation of glycine, proline, lysine carnitine and catecholamine. It facilitates the conversion of cholesterol into bile acids and hence lowers blood cholesterol levels and increases the absorption of iron in the gut by reducing ferric to ferrous state. Finally, it acts as antioxidant, protecting the body from various deleterious effects of free radicals, pollutants and toxins [81]. However, being vitamin $\mathrm{C}$ sensitive to heat and oxygen, it is rapidly oxidized, so much so that its concentration in the Moringa oleifera dried leaves is lower than in the fresh leaves, dropping to 18.7 to $140 \mathrm{mg} / 100 \mathrm{~g}$ of DW $[53,55,56]$.

Difference in (i) environmental conditions in the various origin countries; (ii) genetic of the plant; (iii) drying method [55] and (iv) different extraction and analysis methods, may explain the wide range of vitamin C content in Moringa leaves reported in literature. Freeze-drying seem to better preserve vitamin $\mathrm{C}$ from oxidation, so much so that greater amounts of this vitamin were found in leaves undergone to freeze-drying soon after the collection. In these latter, vitamin $\mathrm{C}$ concentration ranges between 271 and $920 \mathrm{mg} / 100 \mathrm{~g}$ of DW [57,58].

Moringa oleifera fresh leaves are a good source of vitamin E (in particular $\alpha$-tocopherol) and contain approximately $9.0 \mathrm{mg} / 100 \mathrm{~g}$ [59] of this compound, similarly to nuts [80]. Vitamin E acts mainly as liposoluble antioxidants, but it is also involved in the modulation of gene expression, inhibition of cell proliferation, platelet aggregation, monocyte adhesion and regulation of bone mass [82]. Drying procedure determines a concentration of vitamin E up to values of $74.45-122.16 \mathrm{mg} / 100 \mathrm{~g}$ of DW $[53,60,61]$.

Among vitamins of group B, only thiamine, riboflavin and niacin seem present in Moringa oleifera leaves. These vitamins mainly act as cofactors of many enzymes involved in the metabolism of nutrients and energy production, and their concentration in fresh leaves ranges between 0.06 and $0.6 \mathrm{mg} / 100 \mathrm{~g}, 0.05$ and $0.17 \mathrm{mg} / 100 \mathrm{~g}$ and 0.8 and $0.82 \mathrm{mg} / 100 \mathrm{~g}$ for thiamine, riboflavin and niacin, 
respectively $[14,53,60]$, similarly to fruits and vegetable [80]. Only one study reported the contribution of vitamin B1, B2 and B3 of dried leaves of Moringa oleifera [53]. Their concentrations were 2.85, 22.16 and $8.86 \mathrm{mg} / 100 \mathrm{~g}$ of DW, respectively. However, the amount of riboflavin in dried leaves seems very high compared to that of fresh leaves. Further studies are needed to confirm these values. Finally, Girija et al. showed an appreciable physiological availability of these three vitamins in leaves of Moringa oleifera $(61.6 \%, 51.5 \%$ and $39.9 \%$, respectively) [54].

We did not find studies about other vitamin of group B or vitamin D and $\mathrm{K}$ in Moringa oleifera leave; therefore further studies on this topic are needed.

\subsection{Polyphenols}

Moringa oleifera dried leaves are a great source of polyphenols. Their concentrations range from 2090 to $12,200 \mathrm{mgGAE} / 100 \mathrm{~g}$ of DW [63-67] (or 1600 to $3400 \mathrm{mgTAE} / 100 \mathrm{~g}$ of DW) [68,69]. These amounts are greater than those found in fruits and vegetable [83-85]. The different environmental conditions in the various origin countries, the harvesting season [56], the genetic of the plant, the drying method, the leaf maturity stage [63] and the extractive method used [67] may explain such wide range of reported values. Principal polyphenol compounds in Moringa oleifera leaves are flavonoids and phenolic acids.

\subsection{Flavonoids}

Flavonoids are a sub-group of polyphenolic compounds having a benzo- $\gamma$-pyrone structure and are ubiquitous in plants, as they are synthesized in response to microbial infections [86]. Epidemiological studies have consistently shown that high intake of flavonoids has protective effects against many infectious (bacterial and viral diseases) and degenerative diseases such as cardiovascular diseases, cancers, and other age-related diseases [86,87]. Moringa oleifera leaves are an interesting source of flavonoids compounds. Total flavonoids concentration in dried leaves ranges from 5.059 to $12.16 \mathrm{mg} / \mathrm{g}$ of DW [71], namely, close to or larger than that in many fruits and vegetable normally consumed [72,88]. These values are indeed the overall sum of the amounts of single flavonoids. However, some flavonoids were studied only by some authors and, therefore, these amounts may be inaccurate. The total concentration of flavonoids in freeze-dried leaves ranges from 21.0 to $61.62 \mathrm{mgRE} / \mathrm{g}$ of DW [57,58]. Myricetin, quercetin and kaempferol are the main flavonoids found in Moringa oleifera leaves. In dried leaves, myricetin concentration is approximately $5.804 \mathrm{mg} / \mathrm{g}$ of DW, while quercetin and kaempferol concentrations range from 0.207 to $7.57 \mathrm{mg} / \mathrm{g}$ of DW and not detectable amounts (ND) to $4.59 \mathrm{mg} / \mathrm{g}$ of DW, respectively [64-66,71,72,74]. Higher amounts were found in freeze-dried leaves. In particular, quercitin and kaempferol concentrations range from 5.47 to $16.64 \mathrm{mg} / \mathrm{g}$ and 1.5 to $3.5 \mathrm{mg} / \mathrm{g}$ of DW, respectively $[58,73,75]$. Isorhamnetin concentration in dried leaves is approximately $0.118 \mathrm{mg} / \mathrm{g}$ of DW [71], while, in freeze-dried leaves, its concentration is up to 7 times larger, ranging from 0.52 to $0.72 \mathrm{mg} / \mathrm{g}$ of DW [73]. Other flavonoids, such as luteolin, apigenin, daidzein and genistein, were found in not detectable concentrations in Moringa oleifera leaves [65,71]. However these compounds were investigated only in few studies and, therefore, further investigations are needed. In addition, in this case, the high inter-studies variations for these compounds may be explained taking into account 
different environmental conditions, harvesting season, genetic of the plant, drying method, leaf maturity stage, extraction method used and, finally, the different sensitivity of the analytical methods.

\subsection{Phenolic Acids}

Phenolic acids are a sub-group of phenolic compounds derived from hydroxybenzoic acid and hydroxycinnamic acid, naturally present in plants. Thanks to their documented effects on human health, the contribution of food-supplied phenolic acids is a subject of increasing interest. In particular, these compounds are mainly studied for their documented antioxidant, anti-inflammatory, antimutagenic and anticancer properties [89-92]. Particularly abundant in fruit and vegetables, phenolic acids were found in great amounts in Moringa oleifera leaves too. In dried leaves, gallic acid seems to be the most abundant, with a concentration of approximately $1.034 \mathrm{mg} / \mathrm{g}$ of DW $[64,66]$, although Bajpai et al. [65] only found poorly detectable amounts. The concentration of chlorogenic and caffeic acids ranges from 0.018 to $0.489 \mathrm{mg} / \mathrm{g}$ of DW and ND to $0.409 \mathrm{mg} / \mathrm{g}$ of DW, respectively [64-66]. Lower, but appreciable, concentrations were found for ellagic and ferulic acids. Their concentrations range from ND to $0.189 \mathrm{mg} / \mathrm{g}$ and 0.078 to $0.128 \mathrm{mg} / \mathrm{g}$ of DW, respectively [64-66]. Some of these compounds were found more concentrated in freeze-dried leaves. Specifically, Zhang et al. [57], in leaves harvested in Florida and subsequently freeze-dried, found approximately $6.457 \mathrm{mg} / \mathrm{g}$ of DW of o-coumaric acid and $0.536 \mathrm{mg} / \mathrm{g}$ of DW of caffeic acid, while p-coumaric, synaptic, gentistic and syringic acids were found in poorly detectable amounts [57]. Like for the flavonoids, the different environmental conditions, harvesting season, genetic of the plant, drying method, leaf maturity stage, extraction method used and the different sensitivity of the analytical methods may have contributed to the high inter-study variation in the concentrations of phenolic acids in Moringa oleifera leaves.

\subsection{Alkaloids}

Alkaloids are a group of naturally occurring chemical compounds that contain mostly basic nitrogen atoms. This nitrogen may occur in the form of a primary amine $\left(\mathrm{RNH}_{2}\right)$, a secondary amine $\left(\mathrm{R}_{2} \mathrm{NH}\right)$ or a tertiary amine $\left(\mathrm{R}_{3} \mathrm{~N}\right)$. In addition to carbon, hydrogen and nitrogen, most alkaloids contain oxygen [93]. Alkaloids are of particular interest thanks to their pharmacological properties. The presence of these compounds has been confirmed in Moringa oleifera leaves [45,63]. Several of these compounds, such as $N, \alpha$-L-rhamnopyranosyl vincosamide, 4-( $\alpha$-L-rhamnopyranosyloxy) phenylacetonitrile (niazirin), pyrrolemarumine $\quad 4$ "- $O$ - $\alpha$-L-rhamnopyranoside, 4'-hydroxy phenylethanamide- $\alpha$-L-rhamnopyranoside (marumoside A) and its 3-O- $\beta$-D-glucopyranosyl-derivative (marumoside B) and methyl 4-( $\alpha$-L-rhamnopyranosyloxy)-benzylcarbamate, have been isolated in Moringa oleifera leaves [94,95]. However, their amounts in the leaves are still unknown.

\subsection{Glucosinolates and Isothiocyanates}

Glucosinolates are a group of secondary metabolites in plants. Structurally they are $\beta$-S-glucosides of thio-oxime- $O$-sulfates and synthesized from amino acids. Appreciable amounts of these compounds were found in Moringa oleifera leaves. In particular, around 116 and $63 \mathrm{mg} / \mathrm{g}$ of DW in young and 
older leaves, respectively, are reported $[75,96]$. These amounts are close to, and in some case larger than, those found in many cruciferous vegetables (e.g., broccoli, cabbage, radish), mainly sources of these compounds [97]. 4-O-( $\alpha$-L-rhamnopyranosyloxy)-benzyl glucosinolate has been identified as the dominant leaf glucosinolate of Moringa oleifera and is accompanied by lower levels of three isomeric 4-O-( $\alpha$-L-acetylrhamnopyranosyloxy)-benzyl glucosinolates, which reflect the three position of the acetyl group at the rhamnose moiety of the molecule $[75,96]$. The concentrations of these compounds seem affected by the physiological stage of the plant and by the maturity stage of the leaves. The concentration of 4-O-( $\alpha$-L-rhamnopyranosyloxy)-benzyl glucosinolate ranges from 21.84 to $59.4 \mathrm{mg} / \mathrm{g}$ of DW, while the concentrations of the three isomer of 4- $O$-( $\alpha$-L-acetylrhamnopyranosyloxy)-benzyl glucosinolates range from 2.16 to $5.0 \mathrm{mg} / \mathrm{g}$ of DW, 1.2 to $1.8 \mathrm{mg} / \mathrm{g}$ of DW and 12.76 to $50.2 \mathrm{mg} / \mathrm{g}$ of DW for isomer 1, 2 and 3, respectively [73,75]. Amaglo et al. [73] report the presence of 4-hydroxybenzyl (sinalbin), with a concentration ranging between ND and $2.36 \mathrm{mg} / \mathrm{g}$ of DW. Glucosinolates can be hydrolyzed by myrosinase to produce D-glucose and various other degradation products like isothiocyanates [98], which are also present in Moringa oleifera leaves [99,100]. Both glucosinolates and isothiocyanates play an important role in health promoting and prevention of disease [101].

\subsection{Tannins}

Tannins are water-soluble phenolic compounds that bind to and precipitate alkaloids, gelatin and other proteins. They exhibit various biological properties: anti-cancer, antiatherosclerotic, anti-inflammatory, anti-hepatoxic, antibacterial and anti-HIV replication activity [102]. Moringa oleifera leaves are an appreciable source of tannins. Their concentrations range between 13.2 and $20.6 \mathrm{gTAE} / \mathrm{kg}[68,69,76]$ in dried leaves and between 5.0 and $12.0 \mathrm{gTAE} / \mathrm{kg}$ in freeze-dried leaves [77,78]. These amounts are greater than concentrations found in nuts [103], similar to those found in some plants [104] and berries [105], but much lower compared to the concenctrations found in other medicinal plants [106].

\subsection{Saponins}

Saponins are a group of natural compounds that consist of an isoprenoidal-derived aglycone, designated genin or sapogenin, covalently linked to one or more sugar moieties [107]. Even though some saponins have hemolytic side effects, they are studied for their anti-cancer properties $[108,109]$. Moringa oleifera leaves are a good source of saponins. Their concentration in dried leaves is approximately $50 \mathrm{gDE} / \mathrm{kg}$ of DW [69], while in freeze-dried leaves it ranges between 64 and $81 \mathrm{gDE} / \mathrm{kg}$ of DW [77,78]. These amounts are greater than the concentrations found in other plants [106], but slighty lower than ginseng root [110], one of the mainly source of these compounds.

\subsection{Oxalates and Phytates}

Oxalates and phytates are anti-nutritional compounds as they bind minerals inhibiting the intestinal absorption. Moringa oleifera leaves present high contents of these compunds. Oxalates content of dried leaves range from 430 to $1050 \mathrm{mg} / 100 \mathrm{~g}$ of DW [55,76], similar to other plants rich in these compounds [111], while phytates concentration range from 25 to $31 \mathrm{~g} / \mathrm{kg}$ of DW [69] in dried leaves 
and from 21 and $23 \mathrm{~g} / \mathrm{kg}$ of DW in freeze-dried leaves [77,78]. These amounts are greater than those found in legumes and cereals [112,113], but lower than brans [112].

\section{Pharmacology}

\subsection{Antioxidant Properties}

Moringa oleifera leaves are a rich source of antioxidant compounds [114].

Many in vitro studies on antioxidant activity of Moringa oleifera leaves are available in literature [56,58,63-65,67,70,115-119]. Siddhuraju and Becker [58] examined the radical scavenging capacities and antioxidant activities of the aqueous, aqueous methanol, and aqueous ethanol extracts of freeze-dried leaves of Moringa oleifera from different agro-climatic regions. The authors found that different leaves extracts inhibited $89.7 \%-92.0 \%$ peroxidation of linoleic acid and had a scavenging activity on superoxide radicals in a dose-dependent manner $\left(\mathrm{EC}_{50}\right.$ within the range of $0.08-0.2 \mathrm{mg} / \mathrm{mL}$, with the exception of water extract from Indian leaves which has an $\mathrm{EC}_{50}>0.3 \mathrm{mg} / \mathrm{mL}$ ). All of the solvent extracts of leaf samples had a very high radical scavenging activity, however better results were obtained in methanol and ethanol extracts. Both methanol and ethanol extracts of Indian origins showed the highest antioxidant activities, $65.1 \%$ and $66.8 \%$, respectively, in the $\beta$-carotene-linoleic acid system. Nonetheless, increasing concentration of all the extracts had significantly increased reducing power (FRAP). The authors concluded that both methanol $(80 \%)$ and ethanol $(70 \%)$ were the best solvents for the extraction of antioxidant compounds from Moringa oleifera leaves. Similar results were obtained by Vongsak et al. [70] who measured antioxidant activity using various extraction methods on Thai Moringa oleifera leaves. The authors found that the extract obtained macerating dried leaves with $70 \%$ ethanol exhibited high DPPH-scavenging activity $\left(\mathrm{EC}_{50}=62.94 \mathrm{~g} / \mathrm{mL}\right)$ and the highest FRAP value (51.50 mmol $\mathrm{FeSO}_{4}$ equivalents/100 g extract). Iqbal and Bhanger [56] showed that season and agroclimatic locations have profound effect on the antioxidant activity of Moringa oleifera leaves. In this study, the authors collected the leaves in the months of December, March, June, and September from five different areas of Pakistan, and found that the overall antioxidant efficacy was greater in December or March depending upon location, and least in June. The authors suggested that the environmental temperature and, most likely, the soil properties have significant effects on antioxidant activity of Moringa oleifera leaves. The effect of season on antioxidant activity of Moringa oleifera leaves was also found by Shih et al. [115] in leaves collected in Taiwan. The results of this study showed that the winter samples (DPPH-radical scavenging $\mathrm{EC}_{50}=200 \mu \mathrm{g} / \mathrm{mL}$ ) of Moringa oleifera leaves had a significant stronger antioxidant activity than summer samples (DPPH-radical scavenging $\mathrm{EC}_{50}=387 \mu \mathrm{g} / \mathrm{mL}$ ). Finally, Sreelatha and Padma [63] evaluated the antioxidant activity of leaves collected in India in two stages of maturity. The analysis revealed only minor, but significant; differences in the two maturity stages, mature and tender leaves, for the DPPH scavenging activity $\left(\mathrm{EC}_{50}=18.15 v \mathrm{~s} .19 .12 \mu \mathrm{g} / \mathrm{mL}\right)$, superoxide scavenging activity $\left(\mathrm{EC}_{50}=12.71\right.$ vs. $\left.15.51 \mu \mathrm{g} / \mathrm{mL}\right)$, nitric oxide scavenging activity $\left(\mathrm{EC}_{50}=56.77 v s .65 .88 \mu \mathrm{g} / \mathrm{mL}\right)$ and for lipid peroxidation inhibition $\left(\mathrm{EC}_{50}=25.32\right.$ vs. $\left.30.15 \mu \mathrm{g} / \mathrm{mL}\right)$.

Verma et al. [120] examinated the effects of administration of 50 and $100 \mathrm{mg} / \mathrm{kg}$ bw/day of ethyl acetate/polyphenolic extract of Moringa oleifera leaves for 14 days on markers of oxidative stress in 
mice treated with $\mathrm{CCl}_{4}$. The authors observed that the supplementation with Moringa oleifera leaves extract in $\mathrm{CCl}_{4}$-intoxicated rats prevented the increment of lipid peroxide oxidation (LPO) levels, the decrement of glutathione (GSH) concentration and in the activities of superoxide dismutase (SOD) and catalase (CAT) antioxidant enzymes in liver and kidney compared to negative control. Interestingly, the effects obtained in the group treated with $100 \mathrm{mg} / \mathrm{kg} \mathrm{bw} /$ day of leaves extract were comparable to those obtained in the standard group treated with $50 \mathrm{mg} / \mathrm{kg}$ bw/day of vitamin E. Similarly, Luqman et al. [121] studied the effects of administration of increasing amounts of both aqueous and alcoholic extract of Moringa oleifera leaves on markers of oxidative stress in Swiss albino mice. The authors observed an enhancement of GSH concentration in mice erythrocytes treated with the aqueous extract of Moringa oleifera leaves. The observed rise is consistent with the increase in the dose of the extract. Similarly, the dose-responsive effect on LPO was also observed. Moreover, the basal value of malondialdehyde (MDA) concentration was maintained in case of the aqueous extract of Moringa oleifera leaves. Milder, but appreciable, results were obtained using ethanolic extract. Moyo et al. [122] studied the antioxidant properties of diet supplemented with Moringa oleifera dried leaves in goats model. The authors found a significant increment of the activity of GSH in goats supplemented with Moringa leaves as compared with the control group. In comparison, the activities of catalase (CAT) and superoxide dismutase (SOD) of diet supplemented with Moringa oleifera were increased appreciably than the goats fed with ordinary feed. Finally, the supplementation of Moringa oleifera leaves inhibited the amount of MDA generated in liver homogenate. Therefore, also the consumption of Moringa oleifera leaves could protect the animals against diseases induced by oxidative stress. Finally, the administration of Moringa oleifera leaves extract seems to prevent also the oxidative damages caused by high-fat diet. Das et al. [123] reported a lower MDA concentration, a higher GSH concentration and hepatic FRAP in mice fed with high-fat diet and supplemented with Moringa oleifera leaves extract compared to mice only treated with high-fat diet.

Only one study reported the effects of Moringa oleifera leaves extract on oxidative modification of LDL using an "ex vivo" assay [114]. In this study, human plasma was collected and preincubated at $37^{\circ} \mathrm{C}$ for $1 \mathrm{~h}$ with various concentrations of freeze-dried Moringa oleifera leaves extract $(1,10,30$ and $50 \mu \mathrm{g} / \mathrm{mL}$ ). Control group was treated with vitamin $\mathrm{E}$ as standard antioxidant. Oxidation reaction of LDL was initiated by adding freshly prepared $10 \mu \mathrm{M} \mathrm{CuSO}_{4}$ to the samples. This experiment showed that in the presence of Moringa oleifera leaves extract the oxidative modifications of human plasma LDL were significantly inhibited in a dose-dependent manner. In the presence of the leaves extract at a final concentration of $1,10,30$, and $50 \mathrm{~g} / \mathrm{mL}$, the lag-time of conjugated diene formation was increased to 45.0, 151.66, 251.67, and $340.33 \mathrm{~min}$, respectively with an $\mathrm{IC}_{50}$ at $2 \mathrm{~h}$ of $5 \mu \mathrm{g} / \mathrm{mL}$. Moreover, in the presence of Moringa oleifera leaves extract at a final concentration of 1 and $10 \mathrm{~g} / \mathrm{mL}$, the thiobarbituric acid reactive substances (TBARS, products of lipid peroxidation) formation was reduced to 104.16 and $2.80 \mathrm{nmol} / \mathrm{mg}$ LDL protein, respectively. Interestingly, the incubation with higher concentrations of the leaf extract completely blocked TBARS formation. These findings indicate that Moringa oleifera leaves extract could suppress the initiation and propagation of lipid peroxidation also in humans.

In conclusion, many in vitro and in vivo studies have shown antioxidant properties of Moringa oleifera leaves. These findings may be explained by the abundant amounts of antioxidant 
compounds in the leaves. However further studies in human are needed to confirm the results obtained in animals.

\subsection{Anti-Inflammatory and Immunomodulatory Properties}

Inflammation is a protective immunovascular response that involves immune cells, blood vessels, and molecular mediators. The purpose of inflammation is to eliminate the initial cause of cell injury, clear out necrotic cells and tissues damaged from the original insult and the inflammatory process, and to initiate tissue repair.

The anti-inflammatory properties of Moringa oleifera seeds have been so far reported in a number of studies, while only few studies on anti-inflammatory effect of leaves are available in the literature.

Coppin et al. [72] found that 2 on 3 samples of Moringa oleifera leaves were able to inhibit nitric oxide (NO) production by macrophage cells after treatment with bacterial lipopolysaccharide (LPS), whereas the third sample was found not active. The authors attributed this difference to genetics and chemistry of plants which can significantly vary even within the same species. Kooltheat et al. [124] found that the ethyl acetate extract of Moringa oleifera leaves was able to inhibits human macrophage cytokine production (TNF- $\alpha$, IL- 6 and IL-8) induced by extract of cigarette smoke and by LPS, similar to Aspirin (reference drug). Finally, Waterman et al. [100] observed that both Moringa oleifera concentrate and isothiocyanates isolated from the leaves significantly decreased gene expression and production of inflammatory markers in RAW macrophages. Specifically, both attenuated expression of inducible nitric oxide synthase (iNOS), IL-1 $\beta$ and production of NO and TNF $\alpha$ at 1 and $5 \mu \mathrm{M}$.

Sudha et al. [125] and Gupta et al. [126] evaluated the immunomodulatory effect of methanolic and ethanolic extracts of Moringa oleifera leaves, respectively, in cyclophosphamide-induced immunodeficient mice. These authors found that Moringa oleifera leaves extract shown a significant increase in white blood cells, percent neutrophils and serum immunoglobulins, suggesting that it stimulate both cellular and humoral immune response. These results were confirmed by other studies [127-129]. Rajanandh et al. [130] evaluated the anti-inflammatory effects of extract of Moringa oleifera leaves in rats fed with an atherogenic diet in order to induce hyperlipidemia. In this study, rats were fed with 100 and $200 \mathrm{mg} / \mathrm{kg}$ bw of hydro alcoholic extract of Moringa oleifera leaves for a period of 28 days. At the end of the experiment, serum TNF- $\alpha$ and IL-1 were significantly lower in rats treated with both doses of hydro-alcoholic extract of Moringa oleifera leaves compared to control. Recently, Waterman et al. [99] evaluated the effect of supplementation of Moringa oleifera leaves concentrate in obese-induced mice with very high-fat diet (60\% kcal from fat) for 12 weeks. In the experimental group, the very high-fat diet contained 5\% of Moringa oleifera leaves concentrate. The authors observed a reduction of gene expression of pro-inflammatory markers, TNF $\alpha$, IL- 6 and IL-1 $\beta$ in the liver and ileum tissues in mice treated with Moringa oleifera concentrate compared to the control group. Similarly, in adipose tissue, gene expression of $\mathrm{TNF} \alpha$ was reduced while adiponectin had enhanced expression in the treated mice compared to the controls. Finally, Das et al. [131] tested the anti-inflammatory effects of both Moringa oleifera leaves extract and quercitin in mice fed with high-fat diet. The authors found that both Moringa oleifera leaves extract and quercitin inhibited the translocation of p65 subunit of NF-KB (4.2- and 2.3-fold reduction in the expression level, respectively) in comparison with the group only fed with high-fat diet. Moreover, both Moringa oleifera 
leaves extract and quercitin down-regulated the expressions of iNOS, interferon gamma (IFN- $\gamma$ ) and $\mathrm{C}$ reactive protein (CRP) compared to the group only fed with high-fat diet. Finally, release of serum inflammatory cytokines TNF-a and IL-6 potently decreased in the group treated with the extract (30\% and $27 \%$ reduction for TNF-a and IL-6, respectively) and in the group treated with quercitin (27\% and $21 \%$ reduction for TNF-a and IL-6, respectively) in comparison to the HFD group.

In conclusion, many in vitro and in vivo studies in animal model have demonstrated the anti-inflammatory and immunodulatory effects of a supplementation with Moringa oleifera leaves. Many bioactive compounds may be involved in the anti-inflammatory process. Among them, quercetin seems to inhibit activation of NF-kB and also the subsequent NF-kB-dependent downstream events and inflammation [131]. However, many other bioactive compounds naturally present in Moringa oleifera leaves, such as other flavonoids and phenolic acids, may be involved in to anti-inflammatory process. Further studies should be devoted to investigate the potential anti-inflammatory action and the mechanism of action of other bioactive compound naturally present in Moringa oleifera leaves. Concerning immunomodulatory process, the exact mechanism of action of the extract of Moringa oleifera leaves in stimulation of both cellular and the humoral immunity is not clear yet [126]. Therefore, further studies on this subject are needed. Finally, human studies are needed to evaluate the anti-inflammatory and immunomudulatory properties of Moringa oleifera leaves also in human beings.

\subsection{Hypoglycemic Properties}

Hypoglycemic effects of Moringa oleifera leaves are reported in literature [132].

In the study of Ndong et al. [133], male spontaneously diabetic Goto-Kakizaki (GK) rats and non-diabetic male Wistar rats received a single dose of glucose solution and a dose Moringa oleifera leaves (2 g/kg BW and $200 \mathrm{mg} / \mathrm{kg} \mathrm{BW}$, respectively), whereas control groups of both animals only received a single dose of glucose solution. Blood glucose concentration was measured at $0,10,20,30$, 45, 60, 90 and $120 \mathrm{~min}$. Results from OGTT shown that Moringa oleifera significantly decreased blood glucose at 20,30, 45, and $60 \mathrm{~min}$ in GK rats compared to the control and at 10,30 and $45 \mathrm{~min}$ Wistar rats compared to the control after glucose administration. Moreover, in GK rats, the treatment with Moringa oleifera leaves reduced AUC values by $23 \%$, whereas it did not significantly affect these values in control rats. These results suggest that Moringa oleifera has a glucose intolerance ameliorating effect in both GK and Wistar rats, with a greater action in diabetic than in normoglycemic rats. Kar et al. [134] tested hypoglycaemic activity of ethanol extract (95\%) of some Indian medicinal plants, including Moringa oleifera, in alloxan-induced diabetic rats. The authors found that a single dose of $250 \mathrm{mg} / \mathrm{kg} \mathrm{BW}$ of leaves extract determined a halving of serum glucose in a week. In the study of Jaiswal et al. [135], the effect of the aqueous extract of Moringa oleifera leaves on glucose homeostasis was tested in healthy and streptozotocin-induced sub, mild and severely diabetic Wistar rats (STZ, a cytotoxic drug that selectively destroys islet $\beta$ cells). The dose of $200 \mathrm{mg} / \mathrm{kg}$ BW of leaves extract determined a maximum fall of $26.7 \%$ in fasting blood glucose concentration and a maximum fall of $29.9 \%$ in OGGT at $3 \mathrm{~h}$ after glucose administration. The same dose determined a maximum fall of $31.1 \%$ and $32.8 \%$ in OGGT in sub and mild diabetic rats, respectively. Severely diabetic rat were, instead, long treated (21 days) with aqueous extract of Moringa oleifera leaves. The experiment revealed a fall of $25.9 \%, 53.5 \%, 69.2 \%$ in fasting blood glucose at 7,14 and 21 days treatment with 
leaf extract. Interestingly, results obtained in sub, mild and severely diabetic rats were similar to those obtained in Glipizide treated rats $(2.5 \mathrm{mg} / \mathrm{kg} \mathrm{BW}$, reference drug). Similar results were obtained by Edoga et al. [136]. In this study, the aqueous extract produced a dose-dependent reduction in blood glucose levels of normoglycemic and hyperglycemic rats. In normoglycemic rats, the aqueous extract of Moringa oleifera $(100,200$ and $300 \mathrm{mg} / \mathrm{kg}$ ) exhibited a reduction of $23.14 \%, 27.05 \%$ and $33.18 \%$ respectively of the blood glucose levels within 6 hours of administration, while in alloxan-induced diabetic rats the reduction were of $33.29 \%, 40.69 \%$ and $44.06 \%$ respectively. Interestingly, also in this study, similar results were obtained using $200 \mathrm{mg} / \mathrm{kg}$ of tolbutamide (reference drug). Divi et al. [137] tested the antidiabetic properties of aqueous extract of Moringa oleifera leaves in fructose-induced insulin-resistant (IR) and STZ-induced diabetic rats. After administration of $200 \mathrm{mg} / \mathrm{kg} \mathrm{BW}$ of aqueous extract of Moringa oleifera leaves for 60 days the authors observed a decrease in blood glucose concentration in both groups and a decrease of insulin in IR group compared to respectively control. The hypoglycemic effect of aqueous extract of Moringa oleifera leaves in STZ-induced diabetic rats was also confirmed by Yassa et al. [138]. Moreover, in this study histopathological damage of islet cells was also markedly reversed. Moringa oleifera treatment significantly increased the areas of positive purple modified Gomori stained $\beta$-cells (from $60 \%$ to $91 \%$ ) and decreased the area percentage of collagen fibers (from 199\% to 120\%) compared to control values. All these findings were confirmed by other studies using similar approach [139-141].

William et al. [142] examined the effects of Moringa oleifera leaves added to a standardized meal on serum post-prandial glucose concentration at 1 and $2 \mathrm{~h}$ from the consumption, compared to the standard meal alone or a $75 \mathrm{~g}$ oral glucose load in type 2 diabetes mellitus patients. Compared to the glucose load, standard meals with or without vegetable supplements induced a significantly lower post-prandial glucose response as derived from AUCs. However, leaf-supplemented meals caused a lower response $(-21 \%)$ compared to standard meals alone. Moreover, plasma insulin AUCs did not differ significantly between the two meals, suggesting that the hypoglycemic effect of Moringa oleifera leaves supplementation was not due to increased insulin secretion. Kumari [143] examined the hypoglycemic effect of 40-days administration of Moringa oleifera leaves in non-insulin dependent type 2 diabetes mellitus subjects aged 30-60 years old. Recruited subjects were divided in experimental and control group: the first received $8 \mathrm{~g}$ of dried Moringa oleifera leaves for 40 days, whereas the control group didn't receive any treatment. Daily meals were comparable between the two groups in terms of relative food type consumption, nutrients and calories as well. Fasting and post-prandial blood glucose concentrations were taken at baseline and at the end of the experiment. Fasting and post-prandial blood glucose did not differ much from baseline in the control group, while they were significantly reduced in the experimental group ( $-28 \%$ and $-26 \%$, respectively). Finally, Ghiridhari et al. [144] recruited a group of 60 normal weight type 2 diabetes mellitus patients, aged 40-58 years old, on sulfonylurea medication and a standardized calorie-restricted diet (1500 to 1800 Kcal). The patients were equally divided into an experimental and a control groups. Patients in the experimental group were prescribed an unspecified amount of Moringa oleifera leaf for 90 days. The results showed that post prandial blood glucose of experimental group initially was $210 \mathrm{mg} / \mathrm{dL}$ and it reduced to 191,174 and $150 \mathrm{mg} / \mathrm{dL}$ respectively after the first, second and third month of supplementation (with a significant decrease of $9 \%, 17 \%$ and $29 \%$, respectively). In control group post prandial blood glucose level of $179 \mathrm{mg} / \mathrm{dL}$ was substantially maintained during the whole experiment. 
Similar trends were observed for glycated hemoglobin ( $\mathrm{HbA} \mathrm{1c}$ ). In the experimental group initial value of $7.81 \%$ significantly decreased to $7.4 \%$ after the supplementation period, whereas it did not change in the control group. The results indicated that Moringa oleifera leaves are a suitable to reduce the diabetic complications in diabetic patients. However, it should be noted that treatment allocation to patients appear to have not been randomized as baseline values for the two parameters were higher in the experimental group than in the control group, $7.8 \% \pm 0.5 \% v s .7 .4 \% \pm 0.6 \%$ for $\mathrm{HbA}_{1 \mathrm{c}}$ and $210 \pm 49$ vs. $179 \pm 36 \mathrm{mg} / \mathrm{dL}$ for post-prandial glucose response [132]. Finally, it has been suggested that isothicyanates isolated from Moringa oleifera leaves reduced glucose production in liver cells, showing activity at very low concentrations and being close to two orders of magnitude more active than metformin. These compounds were able to decrease phosphoenolpyruvate carboxykinase and glucose-6-phosphatase gene expression suggesting that they act via blocking these rate-limiting steps in liver gluconeogenesis [99].

In conclusion, scientific evidences suggest a potential use of Moringa oleifera leaves in the treatment of diabetes. Many compounds isolated in Moringa oleifera leaves may be involved in the glucose homeostasis. Among theme, isothiocyanates seem to reduce insulin resistance and hepatic gluconeogenesis [99]. However, also polyphenol compounds abundant in Moringa oleifera leaves, such as phenolic acids and flavonoids, may contribute to its effects on glucose homeostasis. These compounds exert, indeed, anti-diabetic effects targeting various cellular signaling pathways in pancreas, liver, skeletal muscle and white adipose tissue. In particular, they influence $\beta$-cell mass and function, as well as energy metabolism and insulin sensitivity in peripheral tissues. Their effects may be due to antioxidant, enzyme inhibition, receptor agonist or antagonist activity or through novel mechanisms yet to be elucidated [145-149]. Phenolic compounds, flavonoids and tannins may be also involved in the ability of Moringa oleifera leaves extract to inhibit the intestinal sucrase and, slightly, the pancreatic $\alpha$-amylase actions [150]. Finally, even though studies on human being highlight the hypoglycemic effects of Moringa oleifera leaves, further larger randomized studies controlled for potential confounders, such as sex, age, race, nutritional status and dietary habits in human are required before using the leaves as herbal drug for the treatment of diabetes.

\subsection{Hypolipidemic Properties}

Hypolipidemic effects of Moringa oleifera leaves are reported in literature [132].

Chumark et al. [114] examined the hypolidipemic effects of Moringa oleifera leaves in rabbits fed with high-cholesterol diet $(5 \%)$ for 12 weeks. A group was concomitantly treated with Moringa oleifera leaves extract $(0.1 \mathrm{~g} / \mathrm{kg} \mathrm{BW} /$ day $)$. At the end of the experiment, rabbits treated with Moringa oleifera leaves presented a total cholesterol, LDL, HDL and triglycerides significantly reduced of $52 \%, 42.7 \%, 44.2 \%$ and $75.4 \%$ respectively, compared to rabbits only fed with high-cholesterol diet, with consequent reduction of $86.52 \%$ of internal carotid atherosclerotic plaque formation. Similar trends were observed in the group treated with $5 \mathrm{mg} / \mathrm{kg} \mathrm{BW}$ of simvastatin (reference drug). The anti-dyslipidemic effects of Moringa oleifera leaves were also examined in rats fed with a high-fat diet [151]. In this study, Wistar rats were fed for 30 days with a high-fat diet containing $20 \%(w / w)$ fat. Animals were divided in two groups, one of which received a daily dose of $1 \mathrm{~g} / \mathrm{kg} \mathrm{BW}$ of aqueous extract of Moringa oleifera leaves. The authors observed a significant lower increasing of 
serum cholesterol in treated compared to untreated rats, but not in liver and kidney. The percentage decrease in serum, liver and kidney was $14.35 \%, 6.40 \%$ and $11.09 \%$, respectively. Similarly, Jain et al. [152], fed albino rats, for 30 days, with a high-fat diet containing $26 \%$ fat. In this case, three groups received different doses of methanolic extracts of Moringa oleifera leaves (150, 300, or $600 \mathrm{mg} / \mathrm{kg} \mathrm{bw} / \mathrm{die}$ ), one group was treated with $4 \mathrm{mg} / \mathrm{kg}$ of simvastatin, whereas another group didn't receive any supplementation. Treatment with methanolic extract, at three different doses significantly decreased the levels of total cholesterol and LDL as compare to the controls. The same was true for VLDL treating rats with the medium and the highest doses of methanolic extracts. Interestingly, the medium and the highest doses of methanolic extracts determined a significant increment of HDL. Similar results were obtained treating rats with simvastatin. In addition, Bais et al. [153] has recently shown that a supplementation of 200 and $400 \mathrm{mg} / \mathrm{kg}$ bw/die of Moringa oleifera leaves extract exhibited anti-obesity effects in high-fat fed mice. Lower food intake was observed in the groups treated with Moringa oleifera leaves extract when compared to the group feed with only high-fat diet. Subsequently, the body weight gain in these groups was significantly lower than the rats feed with only high-fat diet. All these findings were confirmed by other studies using similar approaches [130,153,154].

Other studies evaluated the hypolipidemic effects of Moringa oleifera leaves supplementation in diabetes-induced rats. Diabetes is indeed a risk condition to develop dyslipidemia. Oyedepo et al. [140] evaluated the effect of aqueous extract of Moringa oleifera leaves on plasma total cholesterol, triglycerides, HDL and LDL in alloxan-induced diabetic male rats. Diabetic rats were feed with $400 \mathrm{mg} / \mathrm{kg} \mathrm{BW}$ of aqueous extract of Moringa oleifera leaves for 28 day. At the end of the experiment diabetic rats presented lower plasma total cholesterol, triglycerides and LDL, but not higher HDL, than diabetic rats untreated. Divi et al. [137] observed a decrease of triglycerides $(60.1 \%$ and $56.4 \%)$, total cholesterol (28.8\% and 18.0\%), LDL (31.2\% and 13.9\%) and VLDL (60.4\% and 56.5\%) in both fructose-induced insulin resistant and stz-induced diabetic rats treated with Moringa oleifera leaves extract compared to respectively untreated control. Interestingly, an increment of HDL was observed only in diabetic rats $(40.4 \%)$, but in IR rats in which a decrement $(10.3 \%)$ was observed. Similar results were obtained by other studies [141].

Nambiar et al. [155] firstly examined the potential anti-dyslipidemic effect of Moringa oleifera leaves in 35 hyperlipidemic subjects. Subjects were divided control (18 subjects) and experimental (17 subjects) groups. Groups were similar for anthropometric values (age, height, weight, body mass index, waist/hip ratio) and their daily nutrient intake. The experimental group was treated with $4.4 \mathrm{~g}$ of dehydrated Moringa oleifera leaves, as four $550 \mathrm{mg}$ tablets twice daily, for 50 days. Plasma lipid profiles were determined at the beginning and at the end of the experiment. The authors observed a significant decrease of $1.6 \%$ in plasma total cholesterol and an increment of $6.3 \%$ of HDL in experimental group compared to control group. Not significant trends were observed for LDL, VLDL, and triglycerides. However, the TC/HDL ratio significantly decreased by $6.6 \%$, indicating that the treatment induced a lesser atherogenic lipid profile. Kumari [143] observed a significant decrease of total cholesterol (14\%), LDL (29\%), VLDL (15\%) and triglycerides (14\%) in diabetic subjects treated with $8 \mathrm{~g}$ of Moringa oleifera leaves for 40 days compared to untreated subjects. However, not significant increase of HDL was observed.

In conclusion, scientific evidences suggest a positive effect of Moringa oleifera leaves on lipid homeostasis. Many bioactive compounds may contribute to these effects. It has been suggested that 
phenolic compounds, in particular flavonoids, play important roles on lipid regulation [156]. Moreover, phenolic compounds of Moringa oleifera leaves extract seem to be involved in the inhibition of pancreatic cholesterol esterase activity reducing and delaying the cholesterol absorption, and binding bile acids by forming insoluble complexes and increasing their fecal excretion with theoretical decreasing of plasma cholesterol level [150]. However, studies on this topic are needed to confirm these hypotheses. Moreover, human are still few, and generally conducted on a restricted number of subjects. Therefore, further larger randomized studies controlled for potential confounders, such as sex, age, race, nutritional status and dietary habits in human are required before using the leaves as hypolipidemic and hypocholesterolemic herbal drug.

\subsection{Hepato and Kidney Protective Properties}

Controversial results about the effects of Moringa oleifera leaves on liver and kidney health are reported. Oyagbemi et al. [157] and Asiedu-Gyekye et al. [129] observed an increment in serum alanine aminotransferase (ALT), aspartate aminotransferase (AST), alkaline phosphatase (ALP), blood urea nitrose (BUN) and creatinine following an administration of the extract of Moringa oleifera leaves in mice. Being biomarkers of liver and kidney injury, the authors speculated that leaves might predispose to hepatic and kidney damage. However, histopathological examinations did not reveal any histological lesions in the sinusoids or central vein [129]. On the other hand, other studies [158-161] reported hepatic and kidney protective properties against several drugs, such as isoniazid, rifampicin, pyrazinamide, acetaminophen and gentamicin, attributable to Moringa oleifera leaves. The authors observed a reduction of serum ALT, AST, ALP [158-160] and BUN and creatinine [161] in animals treated with the extract of Moringa oleifera leaves. These findings were confirmed by histological examinations, which revealed an amelioration of the hepatic and kidney damages induced by drugs, in animals treated with Moringa oleifera leaves. Similar results were obtained by Adeyemi and Elebiyo [162] in rats co-treated with Moringa oleifera leaves and $\mathrm{NiSO}_{4}$ in order to induce nephrotoxicity. Finally, Das et al. [123] observed a reduction of ALT, AST and ALP and a lower liver damage in rats fed with high fat diet and co-treated with Moringa oleifera leaves, suggesting a potential role of the leaves in the prevention of nonalcoholic fatty liver disease (NAFLD).

In conclusion, scientific evidences suggest a potential role of Moringa oleifera leaves in the amelioration of the hepatic and kidney damages induced by drugs in animals. However, further studies on human beings are required before using Moringa as herbal medication.

\subsection{Anticancer Properties}

Experimental evidences showed the capacity of Moringa oleifera leaves to protect organism and cell from oxidative DNA damage associated with cancer and degenerative diseases $[163,164]$.

Many in Vitro studies evaluated the anticancer properties of both water and alcoholic extracts of Moringa oleifera leaves on different types of tumor cells lines. Sreelatha et al. [165] found that the aqueous extract of Moringa oleifera leaves exhibited a dose-dependent inhibition of cell proliferation of KB human tumor (KB) cells line. This antiproliferative effect was also associated with an induction of apoptosis, morphological changes and DNA fragmentation. Tiloke et al. [166] observed a significant increment in reactive oxygen species (ROS) with a concomitant decrease in intracellular 
GSH levels caused by a reduction in Nrf2 protein (1.89-fold) and mRNA expression (1.44-fold) in human lung cancer cells treated with Moringa oleifera leaves extract compared to untreated cells. These oxidants can react with DNA in the cell determining a DNA fragmentation with consequent death of cell itself. The pro-apoptotic properties of Moringa oleifera leaves extract were also confirmed by the significant increase in p53 protein (1.02-fold) and mRNA expression (1.59-fold), in caspase-9 (1.28-fold) and caspase-3/7 (1.52-fold) activities and an enhanced expression of Smac/DIABLO in cells treated with the extract. Moringa oleifera leaves extract also caused the cleavage and activation of PARP-1 into 89 and $24 \mathrm{KDa}$ fragments [166]. The apoptosis induction and tumor cell growth inhibition activities of aqueous extract of Moringa oleifera leaves on human lung cancer cells were also studied by Jung [167]. This study confirmed previous results and found that Moringa oleifera leaves extract showed greater cytotoxicity for tumor cells than for normal cells, strongly suggesting that it could be an ideal anticancer therapeutic candidate specific to cancer cells. In the study of Berkovich et al. [168], concentration $\geq 0.75 \mathrm{mg} / \mathrm{mL}$ of Moringa oleifera leaves extract determined a significant inhibition of pancreatic cancer cells (Panc-1) survival as a result of progressive cell apoptosis. In particular, the treatment with $2 \mathrm{mg} / \mathrm{mL}$ Moringa oleifera leaves extract resulted in a reduction of $98 \%$ of Panc-1 cells survival, attributable, at least in part, by a down-regulation of the expression of key NF- $\mathrm{BB}$ signaling pathway proteins. Parvathy and Umamaheshwari [169] found that methanolic extract of Moringa oleifera leaves exhibited less viability on myeloma cells both at highest dose $(2 \%$ at $200 \mu \mathrm{g} / \mathrm{mL})$ and at lowest dose $(12 \%$ at $0.5 \mu \mathrm{g} / \mathrm{mL})$. Khalafalla et al. [170] found that both hot water and ethanolic Moringa oleifera leaves extracts inhibited the viability of acute myeloid leukemia, acute lymphoblastic leukemia and hepatocellular carcinoma cells. On the other hand, all the tested extracts did not exhibit toxic effects against normal mononuclear cells. Charoensin [171] found that dichloromethane extract of Moringa oleifera leaves was more cytotoxic against human hepatocellular carcinoma $\left(\mathrm{HepG} 2 \mathrm{IC}_{50}=120.37 \mu \mathrm{g} / \mathrm{mL}\right)$, colorectal adenocarcinoma (Caco-2; $\mathrm{IC}_{50}=112.46 \mu \mathrm{g} / \mathrm{mL}$ ) and breast adenocarcinoma (MCF-7; $\mathrm{IC}_{50}=133.58 \mu \mathrm{g} / \mathrm{mL}$ ) than methanolic extract $\left(\mathrm{IC}_{50}>250 \mu \mathrm{g} / \mathrm{mL}\right)$. Both extracts had no toxicity on human fibroblast. Finally, Pamok et al. [172] observed that both aqueous and ethanolic extract inhibited cell proliferation of three different types of colon cancer cells lines, with better results using ethanolic extract.

Only one in vivo study is available in literature about anticancer properties of the extract of Moringa oleifera leaves. Purwal et al. [173] studied the effects of oral administration of hydromethanolic and methanolic extracts of Moringa oleifera leaves in murine melanoma tumor model. The authors observed that an oral administration of $500 \mathrm{mg} / \mathrm{kg}$ for 15 days of the extracts determined a delay in the growth of tumors and a significant increase of life span of $48 \%$ and $32 \%$ for methanolic and hydrometanolic extracts respectively.

In conclusion, in vitro studies suggest potential anti-cancer properties of Moringa oleifera leaves. These properties may be explained by the presence of several bioactive compounds, such as 4 -( $\alpha$-L-rhamnosyloxy) benzyl isothiocyanate, niazimicin and $\beta$-sitosterol-3-O- $\beta$-D-glucopyranoside [174,175]. However, further animal studies are needed to confirm these effects. Finally, no studies on human are available in literature. 


\section{Conclusions}

Moringa oleifera is one of the most studied and used plants. Its uses stretch from food and medicinal uses to water purification, biopesticide and production of biodiesel.

Moringa shows diversifications in many features and high morphological variability which may become a resource for the conservation and the selection of Moringa oleifera germplasm. However, some questions still have to be addressed, i.e., collection and characterization of world accessions (both cultivated and natural) and setting a collaborative network among all institutions that already work on Moringa oleifera. This will help scientists and producers to have reliable access to information and materials and better develop Moringa. Moreover, researches focused on the association between phenotypical and molecular data and on genetic maps (both association map and physical map) in order to identify genes are needed within the contest of breeding. Next generation sequencing (NGS) could be an approachable tool to discover genome-wide genetic markers and building saturated genetic map within reasonable cost and time.

Nevertheless Moringa oleifera is an interesting plant for its contribution in bioactive compounds. In particular, leaves, the most used part of the plant, are rich in vitamins, carotenoids, polyphenol, phenolic acids, flavonoids, alkaloids, glucosinolates, isothiocyanates, tannins and saponins. In addition, even if leaves present high variation in the amounts of bioactive compounds as a result of the genetic characteristics of the plant, the environmental conditions to which the plant is subjected and the post-harvest treatments as well, they present greater amounts of these compounds than fruits, vegetables and other plants generally used in human nutrition. On the other hand, the high leaves content of oxalates and phytates could limit the intestinal adsorption of minerals. Therefore, this aspect should be taken in to account for future nutritional researches focused on using Moringa as minerals supplementation.

The high contribution in bioactive compounds may explain the pharmacological properties ascribed to Moringa oleifera leaves. Many in vitro and in vivo studies in animals have widely confirmed numerous pharmacological properties. However, few evidences on human beings are available. Therefore, it is too early to recommend Moringa oleifera leaves as medication in the prevention or treatment of diabetes, cardiovascular disease, dyslipidemia, cancer and infective diseases. Further studies aimed to confirm the pharmacological effects of moringa on human beings and, at the same time, ensuring its safety on human health consequently to a chronic or long-term use should be encouraged.

\section{Conflicts of Interest}

The authors declare no conflict of interest.

\section{References}

1. Paliwal, R.; Sharma, V. A review on horse radish tree (Moringa oleifera): A multipurpose tree with high economic and commercial importance. Asian J. Biotechnol. 2011, 3, 317-328. 
2. Sharma, V.; Paliwal, R.; Sharma, P.; Sharma, S. Phytochemical analysis and evaluation of antioxidant activities of hydro-ethanolic extract of Moringa oleifera Lam. pods. J. Pharm. Res. 2011, 4, 554-557.

3. Roloff, A.; Weisgerber, H.; Lang, U.; Stimm, B. Enzyklopädie der Holzgewächse, Handbuch und Atlas der Dendrologie; WILEY-VCH: Weinheim, Germany, 2009.

4. Ganguly, S. Indian ayurvedic and traditional medicinal implications of indigenously available plants, herbs and fruits: A review. Int. J. Res. Ayurveda Pharm. 2013, 4, 623-625.

5. Mutheeswaran, S.; Pandikumar, P.; Chellappandian, M.; Ignacimuthu, S. Documentation and quantitative analysis of the local knowledge on medicinal plants among traditional Siddha healers in Virudhunagar district of Tamil Nadu, India. J. Ethnopharmacol. 2011, 137, 523-533.

6. Mahmood, K.; Mugal, T.; Haq, I.U. Moringa oleifera: A natural gift-A review. J. Pharm. Sci. Res. 2010, 2, 775-781.

7. Fahey, J.W. Moringa oleifera: A review of the medical evidence for its nutritional, therapeutic, and prophylactic properties. Part 1. Trees Life J. 2005, 1, 1-15.

8. Palada, M.C. Moringa (Moringa oleifera Lam.): A versatile tree crop with horticultural potential in the subtropical United States. HortScience 1996, 31, 794-797.

9. Nouman, W.; Basra, S.M.A.; Siddiqui, M.T.; Yasmeen, A.; Gull, T.; Alcayde, M.A.C. Potential of Moringa oleifera L. as livestock fodder crop: A review. Turk. J. Agric. For. 2014, 38, 1-14.

10. Popoola, J.O.; Obembe, O.O. Local knowledge, use pattern and geographical distribution of Moringa oleifera Lam. (Moringaceae) in Nigeria. J. Ethnopharmacol. 2013, 150, 682-691.

11. Moringa/Moringa Oleifera. Available online: http://www.infonet-biovision.org/default/ct/758/ agroforestry (accessed on 16 April 2015).

12. Ojiako, F.O.; Adikuru, N.C.; Emenyonu, C.A. Critical issues in Investment, Production and Marketing of Moringa oleifera as an Industrial Agricultural raw material in Nigeria. J. Agric. Res. Dev. 2011, 10, 39-56.

13. Morton, J.F. The horseradish tree, Moringa pterygosperma (Moringaceae)-A boon to Arid Lands? Econ. Bot. 1991, 45, 318-333.

14. Ramachandran, C.; Peter, K.V.; Gopalakrishnan, P.K. Drumstick (Moringa oleifera): A multipurpose Indian vegetable. Econ. Bot. 1980, 34, 276-283.

15. Animashaun, J. Prospects of Agriculture Enterprise for Sustainable Economic Development: Success Story of University of Ilorin Moringa Value-Addition Activities. In Proceedings of the 4th International Conference of the African Association of Agricultural Economists, Hammamet, Tunisia, 22-25 September 2013.

16. Jahn, S.A.A.; Musnad, H.A.; Burgstaller, H. The tree that purifies water: Cultivating multipurpose moringaceae in the Sudan. Unasylva 1986, 38, 23-28.

17. Gadzirayi, C.T.; Kubiku, F.N.M.; Mupangwa, J.F.; Mujuru, L.; Chikuvire, T.J. The effect of plant spacing and cutting interval on growth of Moringa oleifera. J. Agric. Sci. Appl. 2013, 2, 131-136.

18. Jiru, D.; Sonder, K.; Alemayehu, L.; Mekonen, Y.; Anjulo, A. Leaf yield and nutritive value of Moringa stenopetala and Moringa oleifera accessions: Its potential role in food security in constrained dry farming agroforestry system. In Proceedings of the Moringa and Other Highly Nutritious Plant Resources: Strategies, Standards and Markets for a Better Impact on Nutrition in Africa, Accra, Ghana, 16-18 November 2006. 
19. Goss, M. A study of the initial establishment of multi-purpose moringa (Moringa oleifera Lam) at various plant densities, their effect on biomass accumulation and leaf yield when grown as vegetable. Afr. J. Plant Sci. 2012, 6, 125-129.

20. Sánchez, N.; Ledin, S.; Ledin, I. Biomass Production and Chemical Composition of Moringa oleifera under different management regimes in Nicaragua. Agrofor. Syst. 2006, 66, 231-242.

21. Ayerza, R. Seed yield components, oil content, and fatty acid composition of two cultivars of moringa (Moringa oleifera Lam.) growing in the Arid Chaco of Argentina. Ind. Crops Prod. 2011, 33, 389-394.

22. Rajangam, J.; Azahakia Manavalan, R.S.; Thangaraj, T.; Vijayakumar, A.; Muthukrishan, N. Status of production and utilisation of Moringa in Southern India. In Proceedings of the International Conference on Development Potential for Moringa Products, Dar es Salaam, Tanzania, 29 October-2 November 2001.

23. Raja, S.; Bagle, B.G.; More, T.A. Drumstick (Moringa oleifera Lamk.) improvement for semiarid and arid ecosystem: Analysis of environmental stability for yield. J. Plant Breed. Crop Sci. 2013, $5,164-170$.

24. Mgendi, M.G.; Nyomora, A.M.; Manoko, M.K. Using morphological markers to assess variations between and within cultivated and non-cultivated provenances of Moringa oleifera Lam. in Tanzania. J. Life Sci. 2011, 5, 387-392.

25. Kumar, A.R.; Prabhu, M.; Ponnuswami, V.; Lakshmanan, V.; Nithyadevi, A. Scientific seed production techniques in moringa. Agric. Rev. 2014, 35, 69-73.

26. Saint Sauveur, A. Moringa exploitation in the world: State of knowledge and challenges. In Proceedings of the Development Potential for Moringa Products, Dar Es Salam, Tanzania, 29 October-2 November 2001.

27. Ohri, D.; Kumar, A. Nuclear DNA amounts in some tropical hardwoods. Caryologia 1986, 39, 303-307.

28. Muluvi, G.M.; Sprent, J.I.; Soranzo, N.; Provan, J.; Odee, D.; Folkard, G.; McNicol, J.W.; Powell, W. Amplified fragment length polymorphism (AFLP) analysis of genetic variation in Moringa oleifera Lam. Mol. Ecol. 1999, 8, 463-470.

29. Muluvi, G.M.; Sprent, J.I.; Odee, D.; Powell, W. Estimates of outcrossing rates in Moringa oleifera using Amplified fragment length polymorphism (AFLP). Afr. J. Biotechnol. 2004, 3, $145-151$.

30. Mgendi, M.; Manoko, M.; Nyomora, A. Genetic diversity between cultivated and non-cultivated Moringa oleifera Lam. provenances assessed by RAPD markers. J. Cell Mol. Biol. 2010, 8, 95-102.

31. Abubakar, B.Y.; Wusirika, R.; MuA'zu, S.; Khan, A.U.; Adamu, A.K. Detection of genetic variability using random amplified polymorphic DNA markers in some accessions of Moringa oleifera Lam. from northern Nigeria. Int. J. Bot. 2011, 7, 237-242.

32. Da Silva, A.V.C.; dos Santos, A.R.F.; Lédo, A.D.S.; Feitosa, R.B.; Almeida, C.S.; da Silva, G.M.; Rangel, M.S.A. Moringa genetic diversity from germplasm bank using RAPD markers. Trop. Subtrop. Agroec. 2012, 15, 31-39. 
33. Saini, R.K.; Saad, K.R.; Ravishankar, G.A.; Giridhar, P.; Shetty, N.P. Genetic diversity of commercially grown Moringa oleifera Lam. cultivars from India by RAPD, ISSR and cytochrome P450-based markers. Plant Syst. Evol. 2013, 299, 1205-1213.

34. Rufai, S.; Hanafi, M.M.; Rafii, M.Y.; Ahmad, S.; Arolu, I.W.; Ferdous, J. Genetic dissection of new genotypes of drumstick tree (Moringa oleifera Lam.) using random amplified polymorphic DNA marker. Biomed. Res. Int. 2013, 2013, 1-6.

35. Popoola, J.; Oluyisola, B.; Obembe, O. Genetic diversity in Moringa Oleifera from nigeria using fruit morpho-metric characters \& random amplified polymorphic DNA (RAPD) markers. Covenant J. Phys. Life Sci. 2014, 1, 43-60.

36. Wu, J.C.; Yang, J.; Gu, Z.J.; Zhang, Y.P. Isolation and characterization of twenty polymorphic microsatellite loci for Moringa oleifera (Moringaceae). HortScience 2010, 45, 690-692.

37. Shahzad, U.; Khan, M.A.; Jaskani, M.J.; Khan, I.A.; Korban, S.S. Genetic diversity and population structure of Moringa oleifera. Conserv. Genet. 2013, 14, 1161-1172.

38. Ganesan, S.K.; Singh, R.; Roy Choudhury, D.; Bharadwaj, J.; Gupta, V.; Singode, A. Genetic diversity and population structure study of drumstick (Moringa oleifera Lam.) using morphological and SSR markers. Ind. Crop. Prod. 2014, 60, 316-325.

39. Haq, S.U.; Jain, R.; Sharma, M.; Kachhwaha, S.; Kothari, S.L. Identification and characterization of microsatellites in expressed sequence tags and their cross transferability in different plants. Int. J. Genomics 2014, 2014, 863948.

40. Davey, J.W.; Hohenlohe, P.A.; Etter, P.D.; Boone, J.Q.; Catchen, J.M.; Blaxter, M.L. Genome-wide genetic marker discovery and genotyping using next-generation sequencing. Nat. Rev. Genet. 2011, 12, 499-510.

41. Sivasankari, B.; Anandharaj, M.; Gunasekaran, P. An ethnobotanical study of indigenous knowledge on medicinal plants used by the village peoples of Thoppampatti, Dindigul district, Tamilnadu, India. J. Ethnopharmacol. 2014, 153, 408-423.

42. Anwar, F.; Latif, S.; Ashraf, M.; Gilani, A.H. Moringa oleifera: A food plant with multiple medicinal uses. Phytother. Res. 2007, 21, 17-25.

43. Abe, R.; Ohtani, K. An ethnobotanical study of medicinal plants and traditional therapies on Batan Island, the Philippines. J. Ethnopharmacol. 2013, 145, 554-565.

44. Yabesh, J.E.; Prabhu, S.; Vijayakumar, S. An ethnobotanical study of medicinal plants used by traditional healers in silent valley of Kerala, India. J. Ethnopharmacol. 2014, 154, 774-789.

45. Kasolo, J.N.; Bimenya, G.S.; Ojok, L.; Ochieng, J.; Ogwal-Okeng, J.W. Phytochemicals and uses of Moringa oleifera leaves in Ugandan rural communities. J. Med. Plant Res. 2010, 4, 753-757.

46. Ashfaq, M.; Basra, S.M.; Ashfaq, U. Moringa: A Miracle Plant for Agro-forestry. J. Agric. Soc. Sci. 2012, 8, 115-122.

47. Karmakar, A.; Karmakar, S.; Mukherjee, S. Properties of various plants and animals feedstocks for biodiesel production. Bioresour. Technol. 2010, 101, 7201-7210.

48. Rashid, U.; Anwar, F.; Moser, B.R.; Knothe, G. Moringa oleifera oil: A possible source of biodiesel. Bioresour. Technol. 2008, 99, 8175-8179.

49. Da Silva, J.P.V.; Serra, T.M.; Gossmann, M.; Wolf, C.R.; Meneghetti, M.R.; Meneghetti, S.M.P. Moringa oleifera oil: Studies of characterization and biodiesel production. Biomass Bioenergy 2010, 34, 1527-1530. 
50. Ofor, M.O.; Nwufo, M.I. The search for alternative energy sources: Jatropha and moringa seeds for biofuel production. J. Agric. Soc. Res. 2011, 11, 87-94.

51. Biswas, W.K.; John, M.B. Life Cycle Assessment of Biodiesel Production from Moringa oleifera Oilseeds; Curtin University of Technology, Centre of Excellence in Cleaner Production (COE): Perth, Australia, 2008.

52. Ferreira, P.M.P.; Farias, D.F.; Oliveira, J.T.D.A.; Carvalho, A.D.F.U. Moringa oleifera: Bioactive compounds and nutritional potential. Rev. Nutr. 2008, 21, 431-437.

53. Price, M.L. The moringa tree. In ECHO Technical Note; ECHO: Myers, FL, USA, 1985.

54. Girija, V.; Sharada, D.; Pushpamma, P. Bioavailability of thiamine, riboflavin and niacin from commonly consumed green leafy vegetables in the rural areas of Andhra Pradesh in India. Int. J. Vitam. Nutr. Res. 1982, 52, 9-13.

55. Joshi, P.; Mehta, D. Effect of dehydration on the nutritive value of drumstick leaves. J. Metabolomics Syst. Biol. 2010, 1, 5-9.

56. Iqbal, S.; Bhanger, M.I. Effect of season and production location on antioxidant activity of Moringa oleifera leaves grown in Pakistan. J. Food Comp. Anal. 2006, 19, 544-551.

57. Zhang, M.; Hettiarachchy, S.N.; Horax, R.; Kannan, A.; Praisoody, M.D.A.; Muhundan, A.; Mallangi, C.R. Phytochemicals, antioxidant and antimicrobial activity of Hibiscus sabdariffa, Centella asiatica, Moringa oleifera and Murraya koenigii leaves. J. Med. Plants Res. 2011, 5, 6672-6680.

58. Siddhuraju, P.; Becker, K. Antioxidant properties of various solvent extracts of total phenolic constituents from three different agroclimatic origins of drumstick tree (Moringa oleifera Lam.) leaves. J. Agric. Food Chem. 2003, 51, 2144-2155.

59. Ching, L.S.; Mohamed, S. Alpha-tocopherol content in 62 edible tropical plants. J. Agric. Food Chem. 2001, 49, 3101-3105.

60. Sanchez-Machado, D.I.; Lopez-Cervantes, J.; Vázquez, N.J.R. High-performance liquid chromatography method to measure $\alpha$-and $\gamma$-tocopherol in leaves, flowers and fresh beans from Moringa oleifera. J. Chromatogr. A 2006, 1105, 111-114.

61. Moyo, B.; Masika, P.J.; Hugo, A.; Muchenje, V. Nutritional characterization of Moringa (Moringa oleifera Lam.) leaves. Afr. J. Biotechnol. 2011, 10, 12925-12933.

62. Kidmose, U.; Yang, R.Y.; Thilsted, S.H.; Christensen, L.P.; Brandt, K. Content of carotenoids in commonly consumed Asian vegetables and stability and extractability during frying. J. Food Comp. Anal. 2006, 19, 562-571.

63. Sreelatha, S.; Padma, P.R. Antioxidant activity and total phenolic content of Moringa oleifera leaves in two stages of maturity. Plant Foods Hum. Nutr. 2009, 64, 303-311.

64. Prakash, D.; Suri, S.; Upadhyay, G.; Singh, B.N. Total phenol, antioxidant and free radical scavenging activities of some medicinal plants. Int. J. Food Sci. Nutr. 2007, 58, 18-28.

65. Bajpai, M.; Pande, A.; Tewari, S.K.; Prakash, D. Phenolic contents and antioxidant activity of some food and medicinal plants. Int. J. Food Sci. Nutr. 2005, 56, 287-291.

66. Singh, B.N.; Singh, B.R.; Singh, R.L.; Prakash, D.; Dhakarey, R.; Upadhyay, G.; Singh, H.B. Oxidative DNA damage protective activity, antioxidant and anti-quorum sensing potentials of Moringa oleifera. Food Chem. Toxicol. 2009, 47, 1109-1116. 
67. Sultana, B.; Anwar, F.; Ashraf, M. Effect of extraction solvent/technique on the antioxidant activity of selected medicinal plant extracts. Molecules 2009, 14, 2167-2180.

68. Bhatta, R.; Saravanan, M.; Baruah, L.; Sampath, K.T. Nutrient content, in vitro ruminal fermentation characteristics and methane reduction potential of tropical tannin-containing leaves. J. Sci. Food Agric. 2012, 92, 2929-2935.

69. Makkar, H.P.S.; Becker, K. Nutrional value and antinutritional components of whole and ethanol extracted Moringa oleifera Leaves. Anim. Feed Sci. Technol. 1996, 63, 211-228.

70. Vongsak, B.; Sithisarn, P.; Mangmool, S.; Thongpraditchote, S.; Wongkrajang, Y.; Gritsanapan, W. Maximizing total phenolics, total flavonoids contents and antioxidant activity of Moringa oleifera Leaf extract by the appropriate extraction method. Ind. Crop. Prod. 2013, 44, 566-571.

71. Yang, R.Y.; Yang, R.Y.; Lin, S.; Kuo, G. Content and distribution of flavonoids among 91 edible plant species. Asia Pac. J. Clin. Nutr. 2008, 17, 275-279.

72. Coppin, J.P.; Xu, Y.; Chen, H.; Pan, M.H.; Ho, C.T.; Juliani, R.; Simon, J.E.; Wu, Q. Determination of flavonoids by LC/MS and anti-inflammatory activity in Moringa oleifera. J. Funct. Foods 2013, 5, 1892-1899.

73. Amaglo, N.K.; Bennett, R.N.; Lo Curto, R.B.; Rosa, E.A.S.; Lo Turco, V.; Giuffrida, A.; Lo Curto, A.; Crea, F.; Timpo, G.M. Profiling selected phytochemicals and nutrients in different tissues of the multipurpose tree Moringa oleifera L., grown in Ghana. Food Chem. 2010, 122, 1047-1054.

74. Sultana, B.; Anwar, F. Flavonols (kaempeferol, quercetin, myricetin) contents of selected fruits, vegetables and medicinal plants. Food Chem. 2008, 108, 879-884.

75. Bennett, R.N.; Mellon, F.A.; Foidl, N.; Pratt, J.H.; Dupont, M.S.; Perkins, L.; Kroon, P.A. Profiling glucosinolates and phenolics in vegetative and reproductive tissues of the multi-purpose trees Moringa oleifera L. (Horseradish Tree) and Moringa stenopetala L. J. Agric. Food Chem. 2003, 51, 3546-3553.

76. Teixeira, E.M.B.; Carvalho, M.R.B.; Neves, V.A.; Silva, M.A.; Arantes-Pereira, L. Chemical characteristics and fractionation of proteins from Moringa oleifera Lam. leaves. Food Chem. 2014, 147, 51-54.

77. Makkar, H.P.S.; Becker, K. Nutrients and antiquality factors in different morphological parts of the Moringa oleifera tree. J. Agric. Sci. 1997, 128, 311-322.

78. Richter, N.; Siddhuraju, P.; Becker, K. Evaluation of nutritional quality of moringa (Moringa oleifera Lam.) leaves as an alternative protein source for Nile tilapia (Oreochromis niloticus L.). Aquaculture 2003, 217, 599-611.

79. Alvarez, R.; Vaz, B.; Gronemeyer, H.; de Lera, A.R. Functions, therapeutic applications, and synthesis of retinoids and carotenoids. Chem. Rev. 2014, 114, 1-125.

80. Gnagnarella, P.; Salvini, S.; Parpinel, M. Food Composition Database for Epidemiological Studies in Italy. Available online: http://www.bda-ieo.it/ (accessed on 16 April 2015).

81. Chambial, S.; Dwivedi, S.; Shukla, K.K.; John, P.J.; Sharma, P. Vitamin C in disease prevention and cure: An overview. Indian J. Clin. Biochem. 2013, 28, 314-328.

82. Borel, P.; Preveraud, D.; Desmarchelier, C. Bioavailability of vitamin E in humans: An update. Nutr. Rev. 2013, 71, 319-331. 
83. Brat, P.; George, S.; Bellamy, A.; Du Chaffaut, L.; Scalbert, A.; Mennen, L.; Arnault, N.; Amiot, M.J. Daily polyphenol intake in France from fruit and vegetables. J. Nutr. 2006, 136, 2368-2373.

84. Cieślik, E.; Gręda, A.; Adamus, W. Contents of polyphenols in fruit and vegetables. Food Chem. 2006, 94, 135-142.

85. Fu, L.; Xu, B.-T.; Xu, X.-R.; Gan, R.-Y.; Zhang, Y.; Xia, E.-Q.; Li, H.-B. Antioxidant capacities and total phenolic contents of 62 fruits. Food Chem. 2011, 129, 345-350.

86. Kumar, S.; Pandey, A.K. Chemistry and biological activities of flavonoids: An overview. Sci. World J. 2013, 2013, 162750.

87. Pandey, K.B.; Rizvi, S.I. Plant polyphenols as dietary antioxidants in human health and disease. Oxid. Med. Cell Longev. 2009, 2, 270-278.

88. Harnly, J.M.; Doherty, R.F.; Beecher, G.R.; Holden, J.M.; Haytowitz, D.B.; Bhagwat, S.; Gebhardt, S. Flavonoid content of U.S. fruits, vegetables, and nuts. J. Agric. Food Chem. 2006, 54, 9966-9977.

89. Verma, S.; Singh, A.; Mishra, A. Gallic acid: Molecular rival of cancer. Environ. Toxicol. Pharmacol. 2013, 35, 473-485.

90. El-Seedi, H.R.; El-Said, A.M.; Khalifa, S.A.; Göransson, U.; Bohlin, L.; Borg-Karlson, A.K.; Verpoorte, R. Biosynthesis, natural sources, dietary intake, pharmacokinetic properties, and biological activities of hydroxycinnamic acids. J. Agric. Food Chem. 2012, 60, 10877-10895.

91. Duthie, G.G.; Wood, A.D. Natural salicylates: Foods, functions and disease prevention. Food Funct. 2011, 2, 515-520.

92. Zhao, Z.; Moghadasian, M.H. Chemistry, natural sources, dietary intake and pharmacokinetic properties of ferulic acid: A review. Food Chem. 2008, 109, 691-702.

93. Cushnie, T.P.T.; Cushnie, B.; Lamb, A.J. Alkaloids: An overview of their antibacterial, antibiotic-enhancing and antivirulence activities. Int. J. Antimicrob. Agents. 2014, 44, 377-386.

94. Panda, S.; Kar, A.; Sharma, P.; Sharma, A. Cardioprotective potential of $N, \alpha$-L-rhamnopyranosyl vincosamide, an indole alkaloid, isolated from the leaves of Moringa oleifera in isoproterenol induced cardiotoxic rats: In vivo and in vitro studies. Bioorg. Med. Chem. Lett. 2013, 23, 959-962.

95. Sahakitpichan, P.; Mahidol, C.; Disadee, W.; Ruchirawat, S.; Kanchanapoom, T. Unusual glycosides of pyrrole alkaloid and 4'-hydroxyphenylethanamide from leaves of Moringa oleifera. Phytochemistry 2011, 72, 791-795.

96. Forster, N.; Ulrichs, C.; Schreiner, M.; Muller, C.T.; Mewis, I. Development of a reliable extraction and quantification method for glucosinolates in Moringa oleifera. Food Chem. 2015, $166,456-464$.

97. Ciska, E.; Martyniak-Przybyszewska, B.; Kozlowska, H. Content of glucosinolates in cruciferous vegetables grown at the same site for two years under different climatic conditions. J. Agric. Food Chem. 2000, 48, 2862-2867.

98. Prakash, D.; Gupta, C. Glucosinolates: The phytochemicals of nutraceutical importance. J. Complement. Integr. Med. 2012, 9, doi:10.1515/1553-3840.1611. 
99. Waterman, C.; Rojas-Silva, P.; Tumer, T.; Kuhn, P.; Richard, A.J.; Wicks, S.; Stephens, J.M.; Wang, Z.; Mynatt, R.; Cefalu, W.; et al. Isothiocyanate-rich Moringa oleifera extract reduces weight gain, insulin resistance and hepatic gluconeogenesis in mice. Mol. Nutr. Food Res. 2015, doi:10.1002/mnfr.201400679.

100. Waterman, C.; Cheng, D.M.; Rojas-Silva, P.; Poulev, A.; Dreifus, J.; Lila, M.A.; Raskin, I. Stable, water extractable isothiocyanates from Moringa oleifera leaves attenuate inflammation in vitro. Phytochemistry 2014, 103, 114-122.

101. Dinkova-Kostova, A.T.; Kostov, R.V. Glucosinolates and isothiocyanates in health and disease. Trends Mol. Med. 2012, 18, 337-347.

102. Kancheva, V.D.; Kasaikina, O.T. Bio-antioxidants-a chemical base of their antioxidant activity and beneficial effect on human health. Curr. Med. Chem. 2013, 20, 4784-805.

103. Venkatachalam, M.; Sathe, S.K. Chemical composition of selected edible nut seeds. J. Agric. Food Chem. 2006, 54, 4705-4714.

104. Johns, T.; Mahunnah, R.L.; Sanaya, P.; Chapman, L.; Ticktin, T. Saponins and phenolic content in plant dietary additives of a traditional subsistence community, the Batemi of Ngorongoro District, Tanzania. J. Ethnopharmacol. 1999, 66, 1-10.

105. Kahkonen, M.P.; Hopia, A.I.; Heinonen, M. Berry phenolics and their antioxidant activity. J. Agric. Food Chem. 2001, 49, 4076-4082.

106. Edeoga, H.O.; Okwu, D.E.; Mbaebie, B.O. Phytochemical constituents of some Nigerian medicinal plants. Afr. J. Biotechnol. 2005, 4, 685-688.

107. Augustin, J.M.; Kuzina, V.; Andersen, S.B.; Bak, S. Molecular activities, biosynthesis and evolution of triterpenoid saponins. Phytochemistry 2011, 72, 435-457.

108. Tian, X.; Tang, H.; Lin, H.; Cheng, G.; Wang, S.; Zhang, X. Saponins: The potential chemotherapeutic agents in pursuing new anti-glioblastoma drugs. Mini Rev Med Chem. 2013, 13, 1709-1724.

109. Man, S.; Gao, W.; Zhang, Y.; Huang, L.; Liu, C. Chemical study and medical application of saponins as anti-cancer agents. Fitoterapia 2010, 81, 703-714.

110. Yun, T.K.; Lee, Y.S.; Kwon, H.Y.; Choi, K.J. Saponin contents and anticarcinogenic effects of ginseng depending on types and ages in mice. Zhongguo Yao Li Xue Bao 1996, 17, 293-298.

111. Gupta, S.; Jyothi Lakshmi, A.; Manjunath, M.N.; Prakash, J. Analysis of nutrient and antinutrient content of underutilized green leafy vegetables. LWT Food Sci. Technol. 2005, 38, 339-345.

112. García-Estepa, R.M.; Guerra-Hernández, E.; García-Villanova, B. Phytic acid content in milled cereal products and breads. Food Res. Int. 1999, 32, 217-221.

113. Hídvégi, M.; Lásztity, R. Phytic acid content of cereals and legumes and interaction with proteins. Chem. Eng. 2003, 46, 59-64.

114. Chumark, P.; Khunawat, P.; Sanvarinda, Y.; Phornchirasilp, S.; Morales, N.P.; Phivthong-Ngam, L.; Ratanachamnong, P.; Srisawat, S.; Pongrapeeporn, K.U. The in vitro and ex vivo antioxidant properties, hypolipidaemic and antiatherosclerotic activities of water extract of Moringa oleifera Lam. leaves. J. Ethnopharmacol. 2008, 116, 439-446.

115. Shih, M.C.; Chang, C.M.; Kang, S.M.; Tsai, M.L. Effect of different parts (leaf, stem and stalk) and seasons (summer and winter) on the chemical compositions and antioxidant activity of Moringa oleifera. Int. J. Mol. Sci. 2011, 12, 6077-6088. 
116. Atawodi, S.E.; Atawodi, J.C.; Idakwo, G.A.; Pfundstein, B.; Haubner, R.; Wurtele, G.; Bartsch, H.; Owen, R.W. Evaluation of the polyphenol content and antioxidant properties of methanol extracts of the leaves, stem, and root barks of Moringa oleifera Lam. J. Med. Food. 2010, 13, 710-716.

117. Shahriar, M.; Hossain, M.I.; Bahar, A.N.M.; Akhter, S.; Haque, M.A.; Bhuiyan, M.A. Preliminary phytochemical screening, in vitro antioxidant and cytotoxic activity of five different extracts of Moringa oleifera Leaf. J. Appl. Pharm. Sci. 2012, 2, 65-68.

118. Pari, L.; Karamac, M.; Kosinska, A.; Rybarczyk, A.; Amarowicz, R. Antioxidant activity of the crude extracts of drumstick tree (Moringa oleifera Lam.) and sweet broomweed (Scoparia dulcis L.) leaves. Pol. J. Food Nutr. Sci. 2007, 57, 203-208.

119. Santos, A.F.S.; Argolo, A.C.C.; Paiva, P.M.G.; Coelho, L.C.B.B. Antioxidant activity of Moringa oleifera tissue extracts. Phytother. Res. 2012, 26, 1366-1370.

120. Verma, A.R.; Vijayakumar, M.; Mathela, C.S.; Rao, C.V. In vitro and in vivo antioxidant properties of different fractions of Moringa oleifera leaves. Food Chem. Toxicol. 2009, 47, 2196-2201.

121. Luqman, S.; Srivastava, S.; Kumar, R.; Maurya, A.K.; Chanda, D. Experimental assessment of Moringa oleifera Leaf and fruit for its antistress, antioxidant, and scavenging potential using in vitro and in vivo assays. Evid. Based Complement. Alternat. Med. 2012, 2012, doi:10.1155/2012/519084.

122. Moyo, B.; Oyedemi, S.; Masika, P.J.; Muchenje, V. Polyphenolic content and antioxidant properties of Moringa oleifera Leaf extracts and enzymatic activity of liver from goats supplemented with Moringa oleifera Leaves/sunflower seed cake. Meat Sci. 2012, 91, 441-447.

123. Das, N.; Sikder, K.; Ghosh, S.; Fromenty, B.; Dey, S. Moringa oleifera Lam. leaf extract prevents early liver injury and restores antioxidant status in mice fed with high-fat diet. Indian J. Exp. Biol. 2012, 50, 404-412.

124. Kooltheat, N.; Sranujit, R.P.; Chumark, P.; Potup, P.; Laytragoon-Lewin, N.; Usuwanthim, K. An ethyl acetate fraction of Moringa oleifera Lam. Inhibits human macrophage cytokine production induced by cigarette smoke. Nutrients 2014, 6, 697-710.

125. Sudha, P.; Asdaq, S.M.; Dhamingi, S.S.; Chandrakala, G.K. Immunomodulatory activity of methanolic leaf extract of Moringa oleifera in animals. Indian J. Physiol. Pharmacol. 2010, 54, $133-140$.

126. Gupta, A.; Gautam, M.K.; Singh, R.K.; Kumar, M.V.; Rao, C.H.V.; Goel, R.K.; Anupurba, S. Immunomodulatory effect of Moringa oleifera Lam. extract on cyclophosphamide induced toxicity in mice. Indian J. Exp. Biol. 2010, 48, 1157-1160.

127. Banji, O.J.F.; Banji, D.; Kavitha, R. Immunomodulatory effects of alcoholic and hydroalcoholic extracts of Moringa olifera Lam leaves. Indian J. Exp. Biol. 2012, 50, 270-276.

128. Gaikwad, S.B.; Mohan, D.G.K.; Reddy, K.J. Moringa oleifera leaves: Immunomodulation in wistar albino rats. Int. J. Pharm. Pharm. Sci. 2011, 3, 426-430.

129. Asiedu-Gyekye, I.J.; Frimpong-Manso, S.; Awortwe, C.; Antwi, D.A.; Nyarko, A.K. Micro- and macroelemental composition and safety evaluation of the nutraceutical Moringa oleifera Leaves. J. Toxicol. 2014, 2014, doi:10.1155/2014/786979.

130. Rajanandh, M.G.; Satishkumar, M.N.; Elango, K.; Suresh, B. Moringa oleifera Lam. A herbal medicine for hyperlipidemia: A pre-clinical report. Asian Pac. J. Trop. Dis. 2012, 2, S790-S795. 
131. Das, N.; Sikder, K.; Bhattacharjee, S.; Majumdar, S.B.; Ghosh, S.; Majumdar, S.; Dey, S. Quercetin alleviates inflammation after short-term treatment in high-fat-fed mice. Food Funct. 2013, 4, 889-898.

132. Mbikay, M. Therapeutic potential of Moringa oleifera Leaves in chronic hyperglycemia and dyslipidemia: A review. Front Pharmacol. 2012, 3, doi:10.3389/fphar.2012.00024.

133. Ndong, M.; Uehara, M.; Katsumata, S.-I.; Suzuki, K. Effects of oral administration of Moringa oleifera Lam on glucose tolerance in goto-kakizaki and wistar rats. J. Clin. Biochem. Nutr. 2007, 40, 229-233.

134. Kar, A.; Choudhary, B.K.; Bandyopadhyay, N.G. Comparative evaluation of hypoglycaemic activity of some Indian medicinal plants in alloxan diabetic rats. J. Ethnopharmacol. 2003, 84, $105-108$.

135. Jaiswal, D.; Kumar Rai, P.; Kumar, A.; Mehta, S.; Watal, G. Effect of Moringa oleifera Lam. leaves aqueous extract therapy on hyperglycemic rats. J. Ethnopharmacol. 2009, 123, 392-396.

136. Edoga, C.O.; Njoku, O.O.; Amadi, E.N.; Okeke, J.J. Blood sugar lowering effect of Moringa oleifera Lam in albino rats. Int. J. Sci. Technol. 2013, 3, 88-90.

137. Divi, S.M.; Bellamkonda, R.; Dasireddy, S.K. Evaluation of antidiabetic and antihyperlipedemic potential of aqueous extract of Moringa oleifera in fructose fed insulin resistant and STZ induced diabetic wistar rats: A comparative study. Asian Asian J. Pharm. Clin. Res. 2012, 5, 67-72.

138. Yassa, H.D.; Tohamy, A.F. Extract of Moringa oleifera leaves ameliorates streptozotocin-induced Diabetes mellitus in adult rats. Acta Histochem. 2014, 116, 844-854.

139. Adeeyo, A.O.; Adefule, A.K.; Ofusori, D.A.; Aderinola, A.A.; Caxton-Martins, E.A. Antihyperglycemic effects of aqueous leaf extracts of mistletoe and Moringa oleifera in streptozotocin-induced diabetes wistar rats. Diabetol. Croat. 2013, 42, 81-88.

140. Oyedepo, T.A.; Babarinde, S.O.; Ajayeoba, T.A. Evaluation of anti-hyperlipidemic effect of aqueous leaves extract of Moringa oleifera in alloxan induced diabetic rats. Int. J. Biochem. Res. Rev. 2013, 3, 162-170.

141. Oparinde, D.P.; Atiba, A.S.; Ajose, O.A.; Eludoyin, A.A.; Adesiyan, A.A. Effect of Moringa oleifera leaf extract on serum lipids and glycaemic control in alloxan induced diabetic albino rats. Int. J. Biomed. Adv. Res. 2014, 5, 519-522.

142. William, F.; Lakshminarayanan, S.; Chegu, H. Effect of some Indian vegetables on the glucose and insulin response in diabetic subjects. Int. J. Food Sci. Nutr. 1993, 44, 191-195.

143. Kumari, D.J. Hypoglycemic effect of Moringa oleifera and Azadirachta indica in type-2 diabetes. Bioscan 2010, 5, 211-214.

144. Arun Giridhari, V.; Malathi, D.; Geetha, K. Anti diabetic property of drumstick (Moringa oleifera) leaf tablets. Int. J. Health Nutr. 2011, 2, 1-5.

145. Babu, P.V.; Liu, D.; Gilbert, E.R. Recent advances in understanding the anti-diabetic actions of dietary flavonoids. J. Nutr. Biochem. 2013, 24, 1777-1789.

146. Oh, Y.S.; Jun, H.S. Role of bioactive food components in diabetes prevention: Effects on Beta-cell function and preservation. Nutr. Metab. Insights. 2014, 7, 51-59.

147. Bahadoran, Z.; Mirmiran, P.; Azizi, F. Dietary polyphenols as potential nutraceuticals in management of diabetes: A review. J. Diabetes. Metab. Disord. 2013, 12, doi:10.1186/22516581-12-43. 
148. Mancuso, C.; Santangelo, R. Ferulic acid: Pharmacological and toxicological aspects. Food Chem. Toxicol. 2014, 65, 185-195.

149. Oboh, G.; Agunloye, O.M.; Adefegha, S.A.; Akinyemi, A.J.; Ademiluyi, A.O. Caffeic and chlorogenic acids inhibit key enzymes linked to type 2 diabetes (in vitro): A comparative study. J. Basic Clin. Physiol. Pharmacol. 2015, 26, 165-170.

150. Adisakwattana, S.; Chanathong, B. Alpha-glucosidase inhibitory activity and lipid-lowering mechanisms of Moringa oleifera Leaf extract. Eur. Rev. Med. Pharmacol. Sci. 2011, 15, 803-808.

151. Ghasi, S.; Nwobodo, E.; Ofili, J.O. Hypocholesterolemic effects of crude extract of leaf of Moringa oleifera Lam in high-fat diet fed wistar rats. J. Ethnopharmacol. 2000, 69, 21-25.

152. Jain, P.G.; Patil, S.D.; Haswani, N.G.; Girase, M.V.; Surana, S.J. Hypolipidemic activity of Moringa oleifera Lam., Moringaceae, on high fat diet induced hyperlipidemia in albino rats. Rev. Bras. Farmacogn. 2010, 20, 969-973.

153. Bais, S.; Singh, G.S.; Sharma, R. Antiobesity and hypolipidemic activity of Moringa oleifera leaves against high fat diet-induced obesity in rats. Adv. Biol. 2014, 2014, 1-9.

154. Tabassum, W.; Kullu, A.R.; Sinha, M.P. Effects of leaf extracts of Moringa oleifera on regulation of hypothyroidism and lipid profile. Bioscan 2013, 8, 665-669.

155. Nambiar, V.S.; Guin, P.; Parnami, S.; Daniel, M. Impact of antioxidants from drumstick leaves on the lipid profile of hyperlipidemics. J. Herb. Med. Toxicol. 2010, 4, 165-172.

156. Siasos, G.; Tousoulis, D.; Tsigkou, V.; Kokkou, E.; Oikonomou, E.; Vavuranakis, M.; Basdra, E.K.; Papavassiliou, A.G.; Stefanadis, C. Flavonoids in atherosclerosis: An overview of their mechanisms of action. Curr. Med. Chem. 2013, 20, 2641-2660.

157. Oyagbemi, A.A.; Omobowale, T.O.; Azeez, I.O.; Abiola, J.O.; Adedokun, R.A.; Nottidge, H.O. Toxicological evaluations of methanolic extract of Moringa oleifera Leaves in liver and kidney of male Wistar rats. J. Basic Clin. Physiol. Pharmacol. 2013, 24, 307-312.

158. Pari, L.; Kumar, N.A. Hepatoprotective activity of Moringa oleifera on antitubercular drug-induced liver damage in rats. J. Med. Food 2002, 5, 171-177.

159. Fakurazi, S.; Hairuszah, I.; Nanthini, U. Moringa oleifera Lam prevents acetaminophen induced liver injury through restoration of glutathione level. Food Chem. Toxicol. 2008, 46, 2611-2615.

160. Sharifudin, S.A.; Fakurazi, S.; Hidayat, M.T.; Hairuszah, I.; Moklas, M.A.; Arulselvan, P. Therapeutic potential of Moringa oleifera extracts against acetaminophen-induced hepatotoxicity in rats. Pharm. Biol. 2013, 51, 279-288.

161. Ouedraogo, M.; Lamien-Sanou, A.; Ramde, N.; Ouédraogo, A.S.; Ouédraogo, M.; Zongo, S.P.; Goumbri, O.; Duez, P.; Guissou, P.I. Protective effect of Moringa oleifera Leaves against gentamicin-induced nephrotoxicity in rabbits. Exp. Toxicol. Pathol. 2013, 65, 335-339.

162. Adeyemi, O.S.; Elebiyo, T.C. Moringa oleifera supplemented diets prevented nickel-induced nephrotoxicity in wistar rats. J. Nutr. Metab. 2014, 2014, 1-8.

163. Sikder, K.; Sinha, M.; Das, N.; Das, D.K.; Datta, S.; Dey, S. Moringa oleifera Leaf extract prevents in vitro oxidative DNA damage. Asian J. Pharm. Clin. Res. 2013, 6, 159-163.

164. Sreelatha, S.; Padma, P.R. Modulatory effects of Moringa oleifera extracts against hydrogen peroxide-induced cytotoxicity and oxidative damage. Hum. Exp. Toxicol. 2011, 30, 1359-1368.

165. Sreelatha, S.; Jeyachitra, A.; Padma, P.R. Antiproliferation and induction of apoptosis by Moringa oleifera Leaf extract on human cancer cells. Food Chem. Toxicol. 2011, 49, 1270-1275. 
166. Tiloke, C.; Phulukdaree, A.; Chuturgoon, A.A. The antiproliferative effect of Moringa oleifera crude aqueous leaf extract on cancerous human alveolar epithelial cells. BMC Complement. Altern. Med. 2013, 13, 226.

167. Jung, I.L. Soluble extract from Moringa oleifera Leaves with a new anticancer activity. PLoS ONE 2014, 9, e95492.

168. Berkovich, L.; Earon, G.; Ron, I.; Rimmon, A.; Vexler, A.; Lev-Ari, S. Moringa Oleifera aqueous leaf extract down-regulates nuclear factor-kappaB and increases cytotoxic effect of chemotherapy in pancreatic cancer cells. BMC Complement. Altern. Med. 2013, 13, doi:10.1186/1472-6882-13-212.

169. Parvathy, M.V.S.; Umamaheshwari, A. Cytotoxic effect of Moringa oleifera leaf extracts on human multiple myeloma cell lines. Trends Med. Res. 2007, 2, 44-50.

170. Khalafalla, M.M.; Abdellatef, E.; Dafalla, H.M.; Nassrallah, A.A.; Aboul-Enein, K.M.; Lightfoot, D.A.; El-Deeb, F.E.; El-Shemy, H.A. Active principle from Moringa oleifera Lam leaves effective against two leukemias and a hepatocarcinoma. Afr. J. Biotechnol. 2010, 9, 8467-8471.

171. Charoensin, S. Antioxidant and anticancer activities of Moringa oleifera leaves. J. Med. Plants Res. 2014, 8, 318-325.

172. Pamok, S.; Saenphet, S.; Vinitketkumnuen, V.; Saenphet, K. Antiproliferative effect of Moringa oleifera Lam. and Pseuderanthemum palatiferum (Nees) Radlk extracts on the colon cancer cells. J. Med. Plant Res. 2011, 6, 139-145.

173. Purwal, L.; Pathak, A.K.; Jain, U.K. In vivo anticancer activity of the leaves and fruits of Moringa oleifera on mouse melanoma. Pharmacologyonline 2010, 1, 655-665.

174. Abdull Razis, A.F.; Ibrahim, M.D.; Kntayya, S.B. Health benefits of Moringa oleifera. Asian Pac. J. Cancer Prev. 2014, 15, 8571-8576.

175. Guevara, A.P.; Vargas, C.; Sakurai, H.; Fujiwara, Y.; Hashimoto, K.; Maoka, T.; Kozuka, M.; Ito, Y.; Tokuda, H.; Nishino, H. An antitumor promoter from Moringa oleifera Lam. Mutat Res. 1999, 440, 181-188.

(C) 2015 by the authors; licensee MDPI, Basel, Switzerland. This article is an open access article distributed under the terms and conditions of the Creative Commons Attribution license (http://creativecommons.org/licenses/by/4.0/). 